\title{
On a degenerate parabolic equation from double phase convection
}

Huashui Zhan ${ }^{1 *}$

"Correspondence:

huashuizhan@163.com

${ }^{1}$ School of Applied Mathematics, Xiamen University of Technology,

Xiamen, Fujian 361024, China

\section{Springer}

\begin{abstract}
The initial-boundary value problem of a degenerate parabolic equation arising from double phase convection is considered. Let $a(x)$ and $b(x)$ be the diffusion coefficients corresponding to the double phase respectively. In general, it is assumed that $a(x)+b(x)>0, x \in \bar{\Omega}$ and the boundary value condition should be imposed. In this paper, the condition $a(x)+b(x)>0, x \in \bar{\Omega}$ is weakened, and sometimes the boundary value condition is not necessary. The existence of a weak solution $u$ is proved by parabolically regularized method, and $u_{t} \in L^{2}\left(Q_{T}\right)$ is shown. The stability of weak solutions is studied according to the different integrable conditions of $a(x)$ and $b(x)$. To ensure the well-posedness of weak solutions, the classical trace is generalized, and that the homogeneous boundary value condition can be replaced by $\left.a(x) b(x)\right|_{x \in \partial \Omega}=0$ is found for the first time.
\end{abstract}

MSC: Primary 35B65; 49N60; secondary 35K10; 35K55

Keywords: Degenerate parabolic equation; Double phase convection; Boundary value condition; Trace

\section{Introduction}

Consider the parabolic equation with a nonlinear convective term

$$
u_{t}=\operatorname{div}\left(a(x)|\nabla u|^{p-2} \nabla u+b(x)|\nabla u|^{q-2} \nabla u\right)+\sum_{i=1}^{N} \frac{\partial f_{i}(u, x, t)}{\partial x_{i}}, \quad(x, t) \in Q_{T},
$$

which arises from the double phase problems, as well as from the flows of incompressible turbulent fluids etc. [3]. In this paper, $Q_{T}=\Omega \times(0, T), \Omega$ is a smooth bounded domain in $\mathbb{R}^{N}, p, q>1, a(x), b(x) \in C(\bar{\Omega}), f_{i}(s, x, t)$ is a Lipschitz function when $|s|$ is bounded.

Though the initial-boundary value problem of the non-Newtonian fluid equation

$$
u_{t}=\operatorname{div}\left(|\nabla u|^{p-2} \nabla u\right), \quad(x, t) \in Q_{T},
$$

has been studied far and widely [12, 13, 24], as a generalized case, equation (1.1) has not provoked researchers' attention until recent years. Since the authors of [20] pointed out that the methods used in studying the well-posedness problem of equation (1.2) are invalid

(c) The Author(s) 2021. This article is licensed under a Creative Commons Attribution 4.0 International License, which permits use sharing, adaptation, distribution and reproduction in any medium or format, as long as you give appropriate credit to the original author(s) and the source, provide a link to the Creative Commons licence, and indicate if changes were made. The images or other third party material in this article are included in the article's Creative Commons licence, unless indicated otherwise in a credit line to the material. If material is not included in the article's Creative Commons licence and your intended use is not permitted by statutory regulation or exceeds the permitted use, you will need to obtain permission directly from the copyright holder. To view a copy of this licence, visit http://creativecommons.org/licenses/by/4.0/. 
for equation (1.1), more and more works related to equation (1.1) have appeared, one can refer to [4-6, 8-10,35]. First of all, let us give a simple review of [20]. If $q \geq p>1$ and

$$
a(x) \geq 0, \quad b(x) \geq 0, \quad a(x)+b(x)>0, \quad x \in \bar{\Omega},
$$

then

$$
a(x)|\nabla u|^{p}+b(x)|\nabla u|^{q} \geq c|\nabla u|^{p},
$$

provided that $|\nabla u| \geq 1$. By its coercivity, we can minimize, with fixed boundary values, the integral

$$
F(u)=\int_{\Omega}\left(a(x)|\nabla u|^{p}+b(x)|\nabla u|^{q}\right) d x
$$

and the local minimizers in the Sobolev class $W_{\text {loc }}^{1, p}(\Omega)$. It is expected (however it is not always true!) that any local minimizer $u$ is also a weak solution to the corresponding Euler's first variation, i.e., the PDE in a divergence form

$$
\sum_{i=1}^{N} \frac{\partial}{\partial x_{i}} a_{i}(x, \nabla u)=0, \quad x \in \Omega,
$$

where $a(x, \nabla u)=\left(a_{i}(x, \nabla u)\right), i=1,2, \ldots, N$, is given by

$$
a(x, \xi)=\left\{p a(x)|\nabla u|^{p-2}+q b(x)|\nabla u|^{q-2}\right\} \nabla u,
$$

satisfying that

$$
|a(x, \xi)| \leq c\left(1+|\xi|^{q-1}\right), \quad \xi \in \mathbb{R}^{N} .
$$

If $u \in W_{\mathrm{loc}}^{1, q}(\Omega)$, we can obtain

$$
|a(x, \nabla u)| \leq M\left(1+|\nabla u|^{q-1}\right) \in L_{\mathrm{loc}}^{\frac{q}{q-1}}(\Omega)=L_{\mathrm{loc}}^{q^{\prime}}(\Omega),
$$

and $u \in W_{\text {loc }}^{1, q}(\Omega)$ would satisfy the (correct) weak form of the equation

$$
\int_{\Omega} \sum_{i=1}^{N} a_{i}(x, \nabla u) \frac{\partial \varphi}{\partial x_{i}} d x=0, \quad \forall \varphi \in W_{0}^{1, q}(\Omega), \operatorname{supp} \varphi \subset \Omega .
$$

But the fact is that a minimizer of the functional (1.3) is only a function of class $u \in$ $W_{\text {loc }}^{1, p}(\Omega)$ ! This is a difference (and a difficulty) with respect to equation (1.2) in which $p=q$. Of course, there is a similar difficulty in the evolution problems. Emphasizing the fact that an evolution problem is usually formulated by a differential equation and not as a minimization, the authors of [20] adopted a different point of view and posed this philosophical question: does a counterpart of the minimization property exist in evolution problems? i.e., may a solution of an evolution problem be a variational minimizer? By introducing a 
new kind of weak solution (called as variational solution in [20]), such a problem has been perfectly solved in [20].

In this paper, we study the well-posedness problem of equation (1.1) only assuming that

$$
\left.[a(x)+b(x)]\right|_{x \in \partial \Omega}=0,\left.\quad[a(x)+b(x)]\right|_{x \in \Omega}>0,
$$

or

$$
\left.[a(x) b(x)]\right|_{x \in \partial \Omega}=0,\left.\quad[a(x) b(x)]\right|_{x \in \partial \Omega}>0 .
$$

Since $a(x) \geq 0, b(x) \geq 0$, on the boundary $\partial \Omega$, if $\left.[a(x)+b(x)]\right|_{x \in \partial \Omega}=0$ implies $\left.[a(x) b(x)]\right|_{x \in \partial \Omega}=0$. But generally, $\left.[a(x) b(x)]\right|_{x \in \partial \Omega}=0$ does not imply $\left.[a(x)+b(x)]\right|_{x \in \partial \Omega}=0$. Let us give some special cases when $N=2, \Omega \subset \mathbb{R}^{2}$ is bounded. For example, we can choose a small constant $\delta<\frac{1}{4}$ and

$$
\Omega_{1}=\left\{x=\left(x_{1}, x_{2}\right): x_{1}^{2}+x_{2}^{2}<1\right\},
$$

$0 \leq a_{1}(x) \in C^{1}\left(\bar{\Omega}_{1}\right)$, and on the boundary it is defined as

$$
a_{1}(x)= \begin{cases}0, & \text { if } x \in\left\{1 \geq x_{2} \geq-\delta, x_{1}^{2}+x_{2}^{2}=1\right\}, \\ \text { smooth connected, } & \text { if } x \in\left\{-\delta \geq x_{2} \geq-2 \delta, x_{1}^{2}+x_{2}^{2}=1\right\}, \\ >0, & \text { if } x \in\left\{-2 \delta>x_{2} \geq-1, x_{1}^{2}+x_{2}^{2}=1\right\} .\end{cases}
$$

While $0 \leq b_{1}(x) \in C^{1}\left(\overline{\Omega_{1}}\right)$, and on the boundary it is defined as

$$
b_{1}(x)= \begin{cases}0, & \text { if } x \in\left\{-1 \leq x_{2} \leq \delta, x_{1}^{2}+x_{2}^{2}=1\right\}, \\ \text { smooth connected, } & \text { if } x \in\left\{2 \delta \geq x_{2} \geq \delta, x_{1}^{2}+x_{2}^{2}=1\right\}, \\ >0, & \text { if } x \in\left\{2 \delta<x_{2} \leq 1, x_{1}^{2}+x_{2}^{2}=1\right\} .\end{cases}
$$

Then $a_{1}(x) b_{1}(x)=0, x \in \partial \Omega_{1}$, but

$$
a_{1}(x)+b_{1}(x)>0, \quad x \in\left\{-2 \delta>x_{2} \geq-1, x_{1}^{2}+x_{2}^{2}=1\right\} \cup\left\{2 \delta<x_{2} \leq 1, x_{1}^{2}+x_{2}^{2}=1\right\} .
$$

So, (1.5) is true, whether or not (1.4) is right.

For a simpler example, let

$$
\Omega_{2}=\left\{x=\left(x_{1}, x_{2}\right): 1<x_{1}^{2}+x_{2}^{2}<4\right\}
$$

and

$$
a_{2}(x)=x_{1}^{2}+x_{2}^{2}-1, \quad b_{2}(x)=4-\left(x_{1}^{2}+x_{2}^{2}\right) .
$$

Clearly, $a_{2}(x)+b_{2}(x)=3>0$ and $a_{2}(x) b_{2}(x)=0$ when $x \in \partial \Omega_{2}$. Also (1.5) is true, whether or not (1.4) is right.

Certainly, if $a(x)=0, b(x)=0$ on the boundary $\partial \Omega$, then conditions (1.4) and (1.5) can be true at the same time. 
We would like to enlarge a bit upon this point. If $a(x)$ and $b(x)$ satisfy

$$
a(x)>0, \quad b(x)>0, \quad x \in \bar{\Omega},
$$

to study the well-posedness of the solutions of equation (1.1), besides the initial value condition

$$
u(x, 0)=u_{0}(x), \quad x \in \Omega,
$$

similar to the usual non-Newtonian fluid equation (1.2), in the sense of the classical trace, the boundary value condition

$$
u(x, t)=0, \quad(x, t) \in \partial \Omega \times(0, T)
$$

should be imposed.

On the other hand, if $a(x)$ and $b(x)$ only satisfy (1.4) or (1.5), equation (1.1) may be degenerate on the boundary $\partial \Omega$, how to define a suitable boundary value condition instead of (1.8) becomes an important problem. In fact, for a degenerate parabolic equation, the weak solution $u(x, t)$ may be too weak to define the trace on the boundary. For example, the authors of [25] pointed out that if the weak solution $u(x, t)$ only has the property

$$
\int_{\Omega} \alpha(x)|\nabla u|^{p} d x<\infty, \int_{\Omega} \alpha(x)^{-\frac{1}{p-1}} d x=+\infty
$$

with that

$$
\alpha(x) \geq 0, \quad x \in \bar{\Omega},
$$

then $C_{0}^{\infty}\left(Q_{T}\right)$ is not dense in the space $\mathbf{B}=\{u: u$ satisfies (1.9) $\}$, and so one cannot define the trace on $\partial \Omega$ in the classical way. The author of [25] gave a new way to deal with the boundary value condition, and we will introduce the related content in the last section of this paper.

In recent years, the author of this paper has been interested in the stability of weak solutions to the following equation:

$$
u_{t}=\operatorname{div}\left(\alpha(x)|\nabla u|^{p(x)-2} \nabla u\right)+f(x, t, u, \nabla u), \quad(x, t) \in Q_{T},
$$

including the special cases of that $p(x)=p$ is a constant, provided that

$$
\alpha(x)=0, \quad x \in \partial \Omega, \quad \alpha(x)>0, \quad x \in \Omega .
$$

If the weak solutions of (1.10) only satisfy (1.9), we also cannot define the trace on the boundary in the classical way. To solve this problem, we have avoided to use the boundary value condition (1.8). Instead, we have found that, to study the uniqueness of weak solution of equation (1.10), condition (1.11) can take place of the boundary value condition (1.8) [26, 29-32].

Actually, for a degenerate parabolic equation, how to deal with the boundary value condition (1.8) has been an important problem for a long time, and there are many papers devoted to this question, one can refer to $[14,15,18,21,27,30]$ etc. for the details. 


\section{The definition of weak solution and the main results}

In the first place, we give some basic concepts.

Assume that $v(x)$ is a positive measurable function defined in $\Omega$. Define the weighted Lebesgue space $L^{p}(v, \Omega), 1<p<\infty$, as the space of all real-valued functions $u$ for which

$$
\|u\|_{p, v}=\left(\int_{\Omega} v(x)|u(x)|^{p} d x\right)^{1 / p}<\infty
$$

Further we suppose that

$$
v(x) \in L_{\mathrm{loc}}^{1}(\Omega), \quad[v(x)]^{-1} \in L_{\mathrm{loc}}^{1 /(p-1)}(\Omega) .
$$

Now, we denote by $W^{1, p}(v, \Omega)$ the space of all real-valued functions $u$ such that the derivative in the sense of distributions fulfills

$$
u \in L^{p}(\Omega) \quad \text { and } \quad v^{1 / p}|\nabla u| \in L^{p}(\Omega)
$$

with the norm

$$
\|u\|_{1, p, v}=\left(\int_{\Omega}|u(x)|^{p} d x+\int_{\Omega} v(x)|\nabla u(x)|^{p} d x\right)^{1 / p} .
$$

By $(2.1)$ we can introduce the subspace $W_{0}^{1, p}(\nu, \Omega)$ of $W^{1, p}(v, \Omega)$ as the closure of $C_{0}^{\infty}(\Omega)$ with respect to the norm (2.2). Moreover, conditions (2.1) imply that $W^{1, p}(\nu, \Omega)$ as well as $W_{0}^{1, p}(v, \Omega)$ are reflexive Banach spaces [19].

Lemma 2.1 Let us suppose that (2.1) holds and

$$
[v(x)]^{-1} \in L^{g^{*}}(\Omega)
$$

with some $g^{*} \geq \frac{1}{p-1}$. Then $W^{1, p}(v, \Omega)$ is continuously imbedded into $W^{1, p_{1}}(\Omega)$, where $p_{1}=$ $\frac{p g^{*}}{g^{*}+1}$.

Remark 2.2 By virtue of compact imbedding theorems (see [6]) and Lemma 2.1, we obtain that the imbedding

$$
W^{1, p}(\nu, \Omega) \rightarrow L^{q}(\Omega)
$$

is compact for $1 \leq q<\frac{N p_{1}}{N-p_{1}}$ if $N>p_{1}$, for $1 \leq q<\infty$ if $N=p_{1}$, for $1 \leq q<\infty$ if $N<p_{1}$. Therefore, if we also suppose that the number $g^{*}$ from Lemma 2.1 satisfies $g^{*}>\frac{N}{p}$. then $W^{1, p}(\mu, \Omega)$ is compactly imbedded into $L^{p}(\Omega)$.

In the second place, we introduce the definition of weak solution.

Definition 2.3 If $u(x, t)$ is a $L^{\infty}\left(Q_{T}\right)$ function, it satisfies

$$
u \in L^{p}\left(0, T ; W^{1, p}(a(x), \Omega)\right) \cap L^{q}\left(0, T ; W^{1, q}(b(x), \Omega)\right), \quad u_{t} \in L^{2}\left(Q_{T}\right)
$$


and

$$
\begin{aligned}
& \iint_{Q_{T}} u_{t} \varphi d x d t \\
& \quad+\iint_{Q_{T}}\left[\left(a(x)|\nabla u|^{p-2} \nabla u+b(x)|\nabla u|^{q-2} \nabla u\right) \nabla \varphi+\sum_{i=1}^{N} f_{i}(u, x, t) \varphi_{x_{i}}\right] d x d t \\
& \quad=0, \quad \forall \varphi \in C_{0}^{1}\left(Q_{T}\right),
\end{aligned}
$$

then $u(x, t)$ is said to be the weak solution of equation (1.1) with the initial boundary values (1.7)-(1.8) provided that

$$
\lim _{t \rightarrow 0} \int_{\Omega}\left(u(x, t)-u_{0}(x)\right) \phi(x) d x=0, \quad \forall \phi(x) \in C_{0}^{\infty}(\Omega)
$$

and the boundary value condition (1.8) is satisfied in the sense of trace.

In the third place, we give the main results.

Theorem 2.4 Suppose that $q \geq p>2, a(x), b(x) \in C(\bar{\Omega})$ satisfies $(1.5), f_{i}(s, x, t)$ is a $C^{1}$ function on $\mathbb{R} \times \bar{Q}_{T}$ satisfies

$$
\left|f_{i}\left(u_{\varepsilon}, x, t\right)\right| \leq c \min \{a(x), b(x)\}, \quad i=1,2, \ldots, N
$$

$u_{0}(x)$ satisfies

$$
u_{0} \in L^{\infty}(\Omega), \quad\left|\nabla u_{0}\right| \in L^{q}(\Omega) .
$$

If

$$
\int_{\Omega} a(x)^{-g_{1} *} d x<\infty, \quad g_{1} * \geq \frac{2}{p-2}
$$

or

$$
\int_{\Omega} b(x)^{-g_{2} *} d x<\infty, \quad g_{2} * \geq \frac{2}{q-2}
$$

then the initial-boundary value problem (1.1)-(1.7)-(1.8) has a solution.

If we do not require $u_{t} \in L^{2}\left(Q_{T}\right)$, instead, $u_{t} \in L^{1}\left(0, T ; W^{-1, q}(\Omega)\right)$ (or a more general Banach space), condition (2.10) or condition (2.11) may not be necessary. Also, condition (2.8) is only used in the proof of the $L^{\infty}$-norm estimate of $u$, and we conjecture it can be replaced by the condition

$$
\sum_{i=1}^{N} \frac{\partial}{\partial x_{i}} f_{i}(\cdot, x, t)<0
$$


In this case, if $u_{0}(x) \geq 0$, then by the maximal value theorem, one may prove that there is a nonnegative weak solution $u(x, t)$ satisfying

$$
0 \leq u(x, t) \leq\left\|u_{0}(x)\right\|_{L^{\infty}(\Omega)} .
$$

Moreover, by considering the minimality for the variational solution, the existence and the regularity of weak solutions was studied in [20] when

$$
2 \leq p \leq q<p+\min \left\{1, \frac{4}{N}\right\} .
$$

However, the main aim of this paper is not to study the existence of weak solution to equation (1.1), we do not pay attention to whether conditions $(2.10)(2.11)$ are optimal or not. Also, we do not try to compare Theorem 2.4 with the results of weak solutions given in [20]. We only give a result on the existence of weak solution for the completeness of the paper. We mainly focus on the stability of weak solutions to equation (1.1) when the coefficients $a(x)$ and $b(x)$ may be degenerate on the boundary $\partial \Omega$.

Theorem 2.5 Let $q \geq p>1, a(x), b(x) \in C(\bar{\Omega})$ satisfy

$$
\int_{\Omega}\left[a(x)^{-\frac{1}{p-1}}+b(x)^{-\frac{1}{q-1}}\right] d x<\infty
$$

$f_{i}(s, x, t)$ be a Lipschitz function when $|s| \leq c, i=1,2, \ldots, N$. If $u(x, t)$ and $v(x, t)$ are two solutions of equation (1.1) with the same homogeneous boundary value condition

$$
u(x, t)=v(x, t)=0, \quad(x, t) \in \partial \Omega \times(0, T),
$$

and with different initial values $u_{0}(x)$ and $v_{0}(x)$ respectively, then

$$
\int_{\Omega}|u(x, t)-v(x, t)| d x \leq \int_{\Omega}\left|u_{0}(x)-v_{0}(x)\right| d x, \quad t \in[0, T) .
$$

If condition (2.13) is invalid, there are three cases

(a)

$$
\int_{\Omega} a(x)^{-\frac{1}{p-1}} d x<\infty, \quad \int_{\Omega} b(x)^{-\frac{1}{q-1}} d x=\infty ;
$$

(b)

$$
\int_{\Omega} a(x)^{-\frac{1}{p-1}} d x=\infty, \quad \int_{\Omega} b(x)^{-\frac{1}{q-1}} d x<\infty
$$

(c)

$$
\int_{\Omega} a(x)^{-\frac{1}{p-1}} d x=\infty, \quad \int_{\Omega} b(x)^{-\frac{1}{q-1}} d x=\infty .
$$

By Proposition 3.3, in cases (a) and (b), we still can impose the boundary value condition (2.14) and obtain stability (2.15). If $a(x), b(x)$ satisfy (c), we cannot impose the boundary 
value condition (2.14) generally. Fortunately, if there are some restrictions between $a(x)$, $b(x)$ and $f_{i}(s, x, t)$, we are still able to prove the following stability of weak solutions without (2.14).

Theorem 2.6 Let $q \geq p>1, a(x), b(x) \in C^{1}(\bar{\Omega})$ satisfy $(1.5), f_{i}(s, x, t)$ be a Lipschitz function when $|s| \leq c$. Suppose that $u(x, t)$ and $v(x)$ are two solutions of $(1.1)$ with the initial values $u_{0}(x)$ and $v_{0}(x)$ respectively. If $a(x), b(x)$ and $f_{i}(s, x, t)$ satisfy

$$
\left|f_{i}(u, x, t)-f_{i}(v, x, t)\right| \leq c\left[a(x)^{\frac{1}{p}}+b(x)^{\frac{1}{q}}\right]|u-v|, \quad i=1,2, \ldots, N,
$$

and for $\eta$ small enough,

$$
\begin{aligned}
& \int_{\Omega \backslash \Omega_{\eta}} a(x)^{1-p}|\nabla a|^{p} d x \leq c, \quad \int_{\Omega \backslash \Omega_{\eta}} a(x) b(x)^{-p}|\nabla b|^{p} d x \leq c, \\
& \int_{\Omega \backslash \Omega_{\eta}} b(x)^{1-q}|\nabla b|^{p} d x \leq c, \quad \int_{\Omega \backslash \Omega_{\eta}} b(x) a(x)^{-q}|\nabla a|^{q} d x \leq c,
\end{aligned}
$$

then stability (2.15) is true.

Here, $\eta$ is a small constant and $\Omega_{\eta}=\{x \in \Omega: a(x) b(x)>\eta\}$.

Theorem 2.7 Let $q \geq p>1, a(x), b(x) \in C^{1}(\bar{\Omega})$ satisfy $(1.4), f_{i}(s, x, t)$ be a Lipschitz function when $|s| \leq c$. Suppose that $u(x, t)$ and $v(x)$ are two solutions of $(1.1)$ with the initial values $u_{0}(x)$ and $v_{0}(x)$, respectively. If $a(x), b(x)$ and $f_{i}(s, x, t)$ satisfy

$$
\begin{aligned}
& \left|f_{i}(u, x, t)-f_{i}(v, x, t)\right| \\
& \quad \leq c\left[(a(x)+b(x))^{\frac{1}{p}}+(a(x)+b(x))^{\frac{1}{q}}\right]|u-v|, \quad i=1,2, \ldots, N,
\end{aligned}
$$

and for $\eta$ small enough,

$$
\begin{aligned}
& \frac{1}{\eta}\left(\int_{\Omega \backslash D_{\eta}}\left|\nabla(a(x)+b(x))^{1+\frac{1}{p}}\right|^{p} d x\right)^{\frac{1}{p}} \leq c, \\
& \frac{1}{\eta}\left(\int_{\Omega \backslash D_{\eta}}\left|\nabla\left(a(x)+b(x)^{1+\frac{1}{q}}\right)\right|^{q} d x\right)^{\frac{1}{q}} \leq c,
\end{aligned}
$$

then stability (2.15) is true.

Here, $\eta$ is a small constant and $D_{\eta}=\{x \in \Omega: a(x)+b(x)>\eta\}$. There is an essential difference between Theorem 2.6 and Theorem 2.7. In Theorem 2.6, $a(x), b(x) \in C^{1}(\bar{\Omega})$ satisfy (1.5), and so

$$
a(x)>0, \quad b(x)>0, \quad x \in \Omega
$$

while in Theorem $2.7 a(x), b(x) \in C^{1}(\bar{\Omega})$ satisfy (1.4), and so

$$
a(x)=0, \quad b(x)=0, \quad x \in \partial \Omega .
$$


But it is possible that there is $x_{0} \in \Omega$,

$$
a\left(x_{0}\right)+b\left(x_{0}\right)>0, \quad a\left(x_{0}\right) b\left(x_{0}\right)=0 .
$$

Naturally, condition (2.16) (or (2.19)) may not be necessary. In the last section of this paper, by giving a generalization of the classical trace of $u \in B V\left(Q_{T}\right)$, we will use a reasonable partial boundary value condition instead of condition (2.16) (or (2.19)) to study the stability of weak solutions.

\section{The existence of weak solutions}

In this section, we want to prove Theorem 2.4. Let us first consider the following CauchyDirichlet problem:

$$
\begin{aligned}
& u_{\varepsilon t}-\operatorname{div}\left((a(x)+\varepsilon)\left(\left|\nabla u_{\varepsilon}\right|^{2}+\varepsilon\right)^{\frac{p-2}{2}} \nabla u_{\varepsilon}+(b(x)+\varepsilon)\left(\left|\nabla u_{\varepsilon}\right|^{2}+\varepsilon\right)^{\frac{q-2}{2}} \nabla u_{\varepsilon}\right) \\
& \quad-\sum_{i=1}^{N} \frac{\partial f_{i}\left(u_{\varepsilon}, x, t\right)}{\partial x_{i}} \\
& \quad=0,(x, t) \in Q_{T}, \\
& u_{\varepsilon}(x, t)=0, \quad(x, t) \in \partial \Omega \times(0, T), \\
& u_{\varepsilon}(x, 0)=u_{\varepsilon 0}(x), \quad x \in \Omega,
\end{aligned}
$$

where $u_{\varepsilon 0} \in C_{0}^{\infty}(\Omega),\left\|u_{\varepsilon 0}\right\|_{L^{\infty}(\Omega)} \leq\left\|u_{0}\right\|_{L^{\infty}(\Omega)},\left|\nabla u_{\varepsilon 0}\right|$ converges to $\left|\nabla u_{0}(x)\right|$ in $L^{q}(\Omega)$.

Since the convection function $f_{i}(s, x, t)$ is a $C^{1}$ function on $\mathbb{R} \times \bar{Q}_{T}, i=1,2, \ldots, N$, by the classical existence theory for parabolic equations [17], similar to [8], we know there is a unique weak solution $u_{\varepsilon} \in C^{0}\left([0, T] ; L^{2}(\Omega)\right) \cap L^{q}\left(0, T ; W_{0}^{1, q}(\Omega)\right)$ with $\partial_{t} u_{\varepsilon} \in$ $L^{q^{\prime}}\left(0, T ; W^{-1, q^{\prime}}(\Omega)\right)$. Now, let us show that

$$
\left\|u_{\varepsilon}\right\|_{L^{\infty}\left(Q_{T}\right)} \leq c
$$

Lemma 3.1 Assume that $a_{1}, b_{1}, \lambda$ are positive constants, where $\lambda>\frac{1}{2}+\frac{b_{1}}{a_{1}}$. Define

$$
\varphi(s)= \begin{cases}e^{\lambda s-1} & s \geq 0 \\ -e^{-\lambda s}+1 & s \leq 0\end{cases}
$$

Then the following properties hold:

1. For any $s \in \mathbb{R}$, we have

$$
|\varphi(s)| \geq \lambda|s|, \quad a_{1} \varphi^{\prime}(s)-b_{1}|\varphi(s)| \geq \frac{a_{1}}{2} e^{\lambda|s|} .
$$

2. For any $s \geq d$, there hold constants $d \geq 0, M>1$, we have

$$
\varphi^{\prime}(s) \leq \lambda M\left[\varphi\left(\frac{s}{l}\right)\right]^{l}, \quad \varphi(s) \leq M\left[\varphi\left(\frac{s}{l}\right)\right]^{l}
$$

where $l>1$ 
3. Let $\Phi(s)=\int_{0}^{s} \varphi(\sigma) d \sigma$. For any $s \geq 0$, if $l>2$, here holds constant $c^{*}>0$, we have

$$
\Phi(s) \geq c^{*}\left[\varphi\left(\frac{s}{l}\right)\right]^{l}
$$

If $1<l<2$, then there exist $d \geq 0$ and $c^{*}=c^{*}(q, d)$ such that

$$
\begin{cases}\Phi(s) \geq c^{*}\left[\varphi\left(\frac{s}{l}\right)\right]^{l}, & \forall s \geq d, \\ \Phi(s) \geq c^{*}\left[\varphi\left(\frac{s}{l}\right)\right]^{2}, & \forall 0 \leq s \leq d .\end{cases}
$$

Lemma 3.1 can be found in [16].

Lemma 3.2 Assume that $u_{\varepsilon}$ is a weak solution of (3.1), then there is a constant $c$ (which is independent of $\varepsilon$ ) such that

$$
\left\|u_{\varepsilon}\right\|_{L^{\infty}\left(Q_{T}\right)} \leq\left\|u_{0}\right\|_{L^{\infty}(\Omega)}+c .
$$

Proof We only give the proof provided that condition (2.10) is true. When condition (2.11) is true, this lemma can be verified in a similar way. Let $k$ be a real number and $\left\|u_{0}\right\|_{L^{\infty}(\Omega)} \leq$ $k$, the function $\varphi$ be defined as (3.4). Define

$$
G_{k}\left(u_{\varepsilon}\right)= \begin{cases}u_{\varepsilon}-k, & u_{\varepsilon}>k, \\ u_{\varepsilon}+k, & u_{\varepsilon}<-k \\ 0, & \left|u_{\varepsilon}\right| \leq k\end{cases}
$$

We can see $\varphi\left(G_{k}\left(u_{\varepsilon}\right)\right) \in V \cap L^{\infty}\left(Q_{T}\right)$. So, for any $\tau \in[0, T]$, we can choose $v=$ $\varphi\left(G_{k}\left(u_{\varepsilon}\right)\right) \chi_{[0, \tau]}$ as a test function (where $\chi_{A}$ is an eigenfunction on the set $A$ ). At the same time, we know that $v_{x_{i}}=\chi_{[0, \tau]} \chi\left\{\left|u_{\varepsilon}\right|>k\right\} \varphi^{\prime}\left(G_{k}\left(u_{\varepsilon}\right)\right) u_{\varepsilon x_{i}}$ and $\nabla v=\chi_{[0, \tau]} \chi\left\{\left|u_{\varepsilon}\right|>\right.$ $k\} \varphi^{\prime}\left(G_{k}\left(u_{\varepsilon}\right)\right) \nabla u_{\varepsilon}$. Since $f_{i}\left(u_{\varepsilon}, x, t\right)$ satisfies $(2.8)$, we have

$$
\begin{aligned}
\int_{0}^{\tau}\left\langle u_{\varepsilon}, \varphi\left(G_{k}\left(u_{\varepsilon}\right)\right)\right\rangle d t & \\
& +\int_{0}^{\tau} \int_{\Omega}\left[(b(x)+\varepsilon)\left(\left|\nabla u_{\varepsilon}\right|^{2}+\varepsilon\right)^{\frac{q-2}{2}}\left|\nabla u_{\varepsilon}\right|^{2}\right. \\
& \left.+(a(x)+\varepsilon)\left(\left|\nabla u_{\varepsilon}\right|^{2}+\varepsilon\right)^{\frac{p-2}{2}}\left|\nabla u_{\varepsilon}\right|^{2}\right] \\
& \cdot \varphi^{\prime}\left(G_{k}\left(u_{\varepsilon}\right)\right) \chi\left\{\left|u_{\varepsilon}\right|>k\right\} d x d t \\
= & -\sum_{i=1}^{N} \int_{0}^{\tau} \int_{\Omega} f_{i}\left(u_{\varepsilon}, x, t\right) \chi\left\{\left|u_{\varepsilon}\right|>k\right\} \varphi^{\prime}\left(G_{k}\left(u_{\varepsilon}\right)\right) u_{\varepsilon x_{i}} d x d t \\
\leq & \int_{0}^{\tau} \int_{\Omega}\left[\frac{1}{p} a(x)\left|\nabla u_{\varepsilon}\right|^{p}+\frac{1}{q} a(x)\right] \chi\left\{\left|u_{\varepsilon}\right|>k\right\} \varphi^{\prime}\left(G_{k}\left(u_{\varepsilon}\right)\right) d x d t,
\end{aligned}
$$

where $\left\langle u_{\varepsilon t}, \varphi\left(G_{k}\left(u_{\varepsilon}\right)\right)\right\rangle$ is the dyadic interaction between $L^{p}\left(0, T ; W_{0}^{1, p}(\Omega)\right)$ and $L^{p^{\prime}}\left(0, T ; W^{-1, p^{\prime}}(\Omega)\right)$. 
Let $A_{k}(t)=\left\{x \in \Omega:\left|u_{\varepsilon}(x, t)\right|>k\right\}$ depend on $k$. We have

$$
\begin{aligned}
\int_{0}^{\tau}\left\langle u_{\varepsilon t}, \varphi\left(G_{k}\left(u_{\varepsilon}\right)\right)\right\rangle d t & =\int_{\Omega} \Phi\left(G_{k}\left(u_{\varepsilon}\right)\right)(\tau) d x-\int_{\Omega} \Phi\left(G_{k}\left(u_{\varepsilon 0}\right)\right) d x \\
& =\int_{A_{k}(\tau)} \Phi\left(G_{k}\left(u_{\varepsilon}\right)\right)(\tau) d x-\int_{A_{k}(0)} \Phi\left(G_{k}\left(u_{\varepsilon 0}\right)\right) d x \\
& =\int_{A_{k}(\tau)} \Phi\left(G_{k}\left(u_{\varepsilon}\right)(\tau) d x .\right.
\end{aligned}
$$

Substituting (3.11) into (3.10), using Lemma 2.1, we can deduce that

$$
\begin{aligned}
& \int_{A_{k}(\tau)} \Phi\left(G_{k}\left(u_{\varepsilon}\right)\right)(\tau) d x+\int_{0}^{\tau} \int_{A_{k}(t)}\left|\nabla u_{\varepsilon}\right|^{p} \varphi^{\prime} d x d t \\
& \leq \int_{A_{k}(\tau)} \Phi\left(G_{k}\left(u_{\varepsilon}\right)\right)(\tau) d x+\int_{0}^{\tau} \int_{A_{k}(t)} a(x)\left|\nabla u_{\varepsilon}\right|^{p} \varphi^{\prime} d x d t \\
& \quad+\int_{0}^{\tau} \int_{A_{k}(t)} b(x)\left|\nabla u_{\varepsilon}\right|^{q} \varphi^{\prime} d x d t \\
& \leq c \int_{A_{k}(\tau)}^{\tau} \Phi\left(G_{k}\left(u_{\varepsilon}\right)\right)(\tau) d x \\
& \quad+c \int_{0}^{\tau} \int_{A_{k}(t)}\left[(b(x)+\varepsilon)\left(\left|\nabla u_{\varepsilon}\right|^{2}+\varepsilon\right)^{\frac{q-2}{2}}\left|\nabla u_{\varepsilon}\right|^{2}\right. \\
&\left.\quad+(a(x)+\varepsilon)\left(\left|\nabla u_{\varepsilon}\right|^{2}+\varepsilon\right)^{\frac{p-2}{2}}\left|\nabla u_{\varepsilon}\right|^{2}\right] \varphi^{\prime} d x d t \\
& \leq c \int_{0}^{\tau} \int_{A_{k}(t)} a(x) \varphi^{\prime}\left(G_{k}\left(u_{\varepsilon}\right)\right) d x d t .
\end{aligned}
$$

Let $\omega_{k}=\varphi\left(\frac{\left|G_{k}\left(u_{\varepsilon}\right)\right|}{p}\right)$. Then

$$
\begin{aligned}
\int_{0}^{\tau} \int_{A_{k}(t)}\left|\nabla u_{\varepsilon}\right|^{p} \varphi^{\prime} d x d t & \geq \frac{1}{2} \int_{0}^{\tau} \int_{A_{k}(t)}\left|e^{\lambda \frac{\left|G_{k}\left(u_{\varepsilon}\right)\right|}{p}} \nabla u\right|^{p} d x d t \\
& =\frac{1}{2} \int_{0}^{\tau} \int_{A_{k}(t)}\left|\frac{p}{\lambda}\right|^{p}\left|\nabla \omega_{k}\right|^{p} d x d t \\
& \geq \frac{1}{2}\left(\frac{1}{\lambda}\right)^{p} \int_{0}^{\tau} \int_{A_{k}(t)}\left|\nabla \omega_{k}\right|^{p} d x d t .
\end{aligned}
$$

By definition we know that $A_{k}(t) \backslash A_{k+d}(t)=\left\{x \in \Omega: k<\left|u_{\varepsilon}(x, t)\right| \leq k+d\right\}$. So, in the set of $A_{k}(t) \backslash A_{k+d}(t)$, we get $0<\left|G_{k}\left(u_{\varepsilon}\right)\right| \leq d, \varphi^{\prime}\left(G_{k}\left(u_{\varepsilon}\right)\right)=\lambda e^{\lambda\left|G_{k}\left(u_{\varepsilon}\right)\right|} \leq \lambda e^{\lambda d}$. Combining (3.6) with (3.12) and (3.13), we have

$$
\begin{aligned}
& \int_{A_{k}(\tau)} \Phi\left(G_{k}\left(u_{\varepsilon}\right)\right)(\tau) d x+\frac{1}{2}\left(\frac{1}{\lambda}\right)^{p} \int_{0}^{\tau} \int_{A_{k}(t)}\left|\nabla \omega_{k}\right|^{p} d x d t \\
& \leq c \lambda \int_{0}^{\tau} \int_{A_{k+d}(t)}\left|w_{k}\right|^{p} d x d t+c \lambda e^{\lambda d} \int_{0}^{\tau} \int_{A_{k}(t) A_{k+d}(t)} d x d t .
\end{aligned}
$$


Since $p \geq 2$, by (3.7), then

$$
\int_{A_{k}(\tau)} \Phi\left(G_{k}\left(u_{\varepsilon}\right)\right)(\tau) d x \geq c^{*} \int_{A_{k}(\tau)}\left|\omega_{k}\right|^{p^{-}} d x
$$

Plugging (3.15) into (3.14) and taking the supremum for $\tau \in\left[0, t_{1}\right]$, with $t_{1} \leq T$ to be determined later, we have

$$
\begin{aligned}
& \sup _{\tau \in\left[0, t_{1}\right]} \int_{A_{k}(\tau)}\left|\omega_{k}\right|^{p^{-}} d x+\frac{1}{2}\left(\frac{1}{\lambda}\right)^{p} \int_{0}^{t_{1}} \int_{A_{k}(t)}\left|\nabla \omega_{k}\right|^{p} d x d t \\
& \quad \leq c \lambda \int_{0}^{\tau} \int_{A_{k+d}(t)}\left|w_{k}\right|^{p} d x d t+c_{1} \lambda e^{\lambda d} \int_{0}^{\tau} \int_{A_{k}(t) A_{k+d}(t)} d x d t .
\end{aligned}
$$

Let $\psi_{k}=\int_{0}^{t_{1}} \mu\left(A_{k}(t)\right) d t$. By choosing

$$
c_{1}\left(t_{1} \mu(\Omega)\right)^{\frac{p}{N+p}} \leq \frac{1}{2}
$$

where $\mu(\Omega)$ is the Lebesgue measure of $\Omega$. Now, using the embedding inequality $[16,24]$, we can deduce that

$$
\left(\int_{0}^{t_{1}} \int_{A_{k}(t)}\left|\omega_{k}\right|^{p \frac{N+p}{N}} d x d t\right)^{\frac{N}{N+p}} \leq \gamma\left(\sup _{\tau \in\left[0, t_{1}\right]} \int_{A_{k}(\tau)}\left|\omega_{k}\right|^{p} d x+\int_{0}^{t_{1}} \int_{A_{k}(t)}|\nabla \omega|^{p} d x d t\right)
$$

where $\gamma$ is a constant independent of $t_{1}$, similar to the proof of Theorem 2.2 in [16], it follows from (3.16) that

$$
\psi_{l} \leq \frac{c}{(l-k)^{\frac{p(N+p)}{N}}} \psi_{k}^{\left(1-\frac{1}{r}\right) \frac{N+p}{N}}
$$

where $r>\frac{N+p}{N}$ is a constant, and so

$$
\left(1-\frac{1}{r}\right) \frac{N+p}{N}>1
$$

Therefore, thanks to the iteration lemma in [21], from (3.18), we eventually obtain that

$$
\psi_{\left(\left\|u_{0}\right\|_{L} \infty(\Omega)+D\right)}=0
$$

where $D>0$ is a constant depending only on $p, N, t_{1}, r, \Omega$. This proves that, for fixed $\lambda$ validating Lemma 2.1 ,

$$
\|u(x, t)\|_{L^{\infty}\left(Q_{t_{1}}\right)} \leq\left\|u_{0 \varepsilon}\right\|_{L^{\infty}(\Omega)}+D
$$

Finally, we partition the time interval $[0, T]$ into finite subintervals $\left[0, t_{1}\right],\left[t_{1}, t_{2}\right], \ldots$, $\left[t_{n-1}, T\right]$ such that the conditions similar to (3.17) are available for each subinterval $\left[t_{i}, t_{i+1}\right]$, then we deduce an inequality of the form (3.19). Eventually, we have conclusion (3.9). 
Proof of Theorem 2.4 Multiplying (3.1) by $u_{\varepsilon}$ and integrating it over $Q_{T}$ yield

$$
\begin{aligned}
& \frac{1}{2} \int_{\Omega} u_{\varepsilon}^{2} d x+\iint_{Q_{T}}\left[(a(x)+\varepsilon)\left(\left|\nabla u_{\varepsilon}\right|^{2}+\varepsilon\right)^{\frac{p-2}{2}}\left|\nabla u_{\varepsilon}\right|^{2}\right. \\
& \left.+(b(x)+\varepsilon)\left(\left|\nabla u_{\varepsilon}\right|^{2}+\varepsilon\right)^{\frac{q-2}{2}}\left|\nabla u_{\varepsilon}\right|^{2}\right] d x d t \\
& \quad+\sum_{i=1}^{N} \iint_{Q_{T}} \frac{\partial f_{i}\left(u_{\varepsilon}, x, t\right)}{\partial x_{i}} u_{\varepsilon} d x d t \\
& =\frac{1}{2} \int_{\Omega} u_{\varepsilon 0}^{2} d x .
\end{aligned}
$$

Since $f_{i}(s, x, t)$ is a Lipschitz function when $|s| \leq c, \frac{\partial f_{i}(s, x, t)}{\partial s}$ exists almost everywhere and is bounded. If $\int_{\Omega} a(x)^{-\frac{2}{p-2}} d x<\infty$, then $\int_{\Omega} a(x)^{-\frac{1}{p-1}} d x<\infty$, and

$$
\begin{aligned}
\left|\int_{\Omega} \frac{\partial f_{i}\left(u_{\varepsilon}, x, t\right)}{\partial x_{i}} u_{\varepsilon} d x\right| & \leq \int_{\Omega}\left|\frac{\partial f_{i}(s, x, t)}{\partial s}\right|_{s=u_{\varepsilon}} u_{\varepsilon x_{i}}|| u_{\varepsilon} \mid d x+c \\
& \leq c \int_{\Omega}\left|\frac{\partial f_{i}(s, x, t)}{\partial s}\right|_{s=u_{\varepsilon}} u_{\varepsilon x_{i}} \mid d x+c \\
& \leq \frac{1}{4} \int_{\Omega} a(x)\left|\nabla u_{\varepsilon}\right|^{p} d x+c \int_{\Omega} a(x)^{-\frac{1}{p-1}} d x+c \\
& \leq \frac{1}{4} \int_{\Omega} a(x)\left|\nabla u_{\varepsilon}\right|^{p} d x+c .
\end{aligned}
$$

Or similarly, if $\int_{\Omega} b(x)^{-\frac{2}{q-2}} d x<\infty$, we know that $\int_{\Omega} b(x)^{-\frac{1}{q-1}} d x<\infty$ and

$$
\begin{aligned}
\left|\int_{\Omega} \frac{\partial f_{i}\left(u_{\varepsilon}, x, t\right)}{\partial x_{i}} u_{\varepsilon} d x\right| & \leq \int_{\Omega}\left|\frac{\partial f_{i}(s, x, t)}{\partial s}\right|_{s=u_{\varepsilon}} u_{\varepsilon x_{i}}|| u_{\varepsilon} \mid d x+c \\
& \leq c \int_{\Omega}\left|\frac{\partial f_{i}(s, x, t)}{\partial s}\right|_{s=u_{\varepsilon}} u_{\varepsilon x_{i}} \mid d x+c \\
& \leq \frac{1}{4} \int_{\Omega} b(x)\left|\nabla u_{\varepsilon}\right|^{q} d x+c \int_{\Omega} b(x)^{-\frac{1}{q-1}} d x+c \\
& \leq \frac{1}{4} \int_{\Omega} b(x)\left|\nabla u_{\varepsilon}\right|^{q} d x+c .
\end{aligned}
$$

Accordingly, based on condition (2.10) or condition (2.10), by (3.20), we obtain

$$
\begin{aligned}
\int_{\Omega} u_{\varepsilon}^{2} d x+\iint_{Q_{T}} a(x)\left|\nabla u_{\varepsilon}\right|^{p} d x d t+\iint_{Q_{T}} b(x)\left|\nabla u_{\varepsilon}\right|^{q} d x d t \\
\leq \int_{\Omega} u_{\varepsilon}^{2} d x+\iint_{Q_{T}}(a(x)+\varepsilon)\left(\left|\nabla u_{\varepsilon}\right|^{2}+\varepsilon\right)^{\frac{p-2}{2}}\left|\nabla u_{\varepsilon}\right|^{2} d x d t \\
\quad+\iint_{Q_{T}}(b(x)+\varepsilon)\left(\left|\nabla u_{\varepsilon}\right|^{2}+\varepsilon\right)^{\frac{q-2}{2}}\left|\nabla u_{\varepsilon}\right|^{2} d x d t
\end{aligned}
$$

$\leq c$. 
Let $\Omega_{1} \subset \subset \Omega$. Then there exists a constant $c\left(\Omega_{1}\right)$ such that

$$
a(x) \geq c\left(\Omega_{1}\right)>0, \quad b(x) \geq c\left(\Omega_{1}\right)>0 .
$$

By $q \geq p>2$, (3.21) yields

$$
\begin{aligned}
& \int_{0}^{T} \int_{\Omega_{1}}\left|\nabla u_{\varepsilon}\right|^{2} d x d t \\
& \quad \leq c\left(\int_{0}^{T} \int_{\Omega_{1}}\left|\nabla u_{\varepsilon}\right|^{p} d x d t\right)^{\frac{2}{p}} \\
& \quad \leq c\left(\Omega_{1}\right)\left[\left(\int_{0}^{T} \int_{\Omega_{1}} a(x)\left|\nabla u_{\varepsilon}\right|^{p} d x d t\right)^{\frac{2}{p}}+\left(\int_{0}^{T} \int_{\Omega_{1}} b(x)\left|\nabla u_{\varepsilon}\right|^{q} d x d t\right)^{\frac{2}{q}}\right] \\
& \quad \leq c .
\end{aligned}
$$

Multiplying (2.9) by $u_{\varepsilon t}$, we have

$$
\begin{aligned}
& \iint_{Q_{T}}\left|u_{\varepsilon t}\right|^{2} d x d t \\
& =\sum_{i=1}^{N} \iint_{Q_{T}} \frac{\partial}{\partial x_{i}}\left[(a(x)+\varepsilon)\left(\left|\nabla u_{\varepsilon}\right|^{2}+\varepsilon\right)^{\frac{p-2}{2}} u_{\varepsilon x_{i}}\right. \\
& \left.\quad+(b(x)+\varepsilon)\left(\left|\nabla u_{\varepsilon}\right|^{2}+\varepsilon\right)^{\frac{q-2}{2}} u_{\varepsilon x_{i}}\right] u_{\varepsilon t} d x d t \\
& \quad+\sum_{i=1}^{N} \iint_{Q_{T}} u_{\varepsilon t} \frac{\partial f_{i}\left(u_{\varepsilon}, x, t\right)}{\partial x_{i}} d x d t
\end{aligned}
$$

For every term in (1.7), firstly, we have

$$
\begin{aligned}
& \sum_{i=1}^{N} \iint_{Q_{T}} \frac{\partial}{\partial x_{i}}\left((a(x)+\varepsilon)\left(\left|\nabla u_{\varepsilon}\right|^{2}+\varepsilon\right)^{\frac{p-2}{2}} u_{\varepsilon x_{i}}\right) u_{\varepsilon t} d x d t \\
& \quad=-\sum_{i=1}^{N} \iint_{Q_{T}}(a(x)+\varepsilon)\left(\left|\nabla u_{\varepsilon}\right|^{2}+\varepsilon\right)^{\frac{p-2}{2}} u_{\varepsilon x_{i}} u_{\varepsilon x_{i} t} d x d t \\
& \quad=-\frac{1}{2} \sum_{i=1}^{N} \iint_{Q_{T}}(a(x)+\varepsilon) \frac{d}{d t} \int_{0}^{\left|\nabla u_{\varepsilon}\right|^{2}+\varepsilon} s^{\frac{p-2}{2}} d s d x d t
\end{aligned}
$$

and

$$
\begin{aligned}
& \sum_{i=1}^{N} \iint_{Q_{T}} \frac{\partial}{\partial x_{i}}\left((b(x)+\varepsilon)\left(\left|\nabla u_{\varepsilon}\right|^{2}+\varepsilon\right)^{\frac{q-2}{2}} u_{\varepsilon x_{i}}\right) u_{\varepsilon t} d x d t \\
& =-\sum_{i=1}^{N} \iint_{Q_{T}}(b(x)+\varepsilon)\left(\left|\nabla u_{\varepsilon}\right|^{2}+\varepsilon\right)^{\frac{q-2}{2}} u_{\varepsilon x_{i}} u_{\varepsilon x_{i} t} d x d t \\
& \quad=-\frac{1}{2} \sum_{i=1}^{N} \iint_{Q_{T}}(b(x)+\varepsilon) \frac{d}{d t} \int_{0}^{\left|\nabla u_{\varepsilon}\right|^{2}+\varepsilon} s^{\frac{q-2}{2}} d s d x d t
\end{aligned}
$$


Secondly, if $\int_{\Omega} a(x)^{-\frac{2}{p-2}}(x) d x<\infty$, by the Hölder inequality, we have

$$
\begin{aligned}
& \iint_{Q_{T}}\left|\nabla u_{\varepsilon}\right|^{2} d x d t \\
& \quad=c \iint_{Q_{T}} a(x)^{-\frac{2}{p}} a(x)^{\frac{2}{p}}\left|\nabla u_{\varepsilon}\right|^{2} d x d t \\
& \quad \leq c\left(\iint_{Q_{T}} a(x)^{-\frac{2}{p-2}} d x d t\right)^{\frac{p-2}{p}}\left(\iint_{Q_{T}} a(x)\left|\nabla u_{\varepsilon}\right|^{p} d x d t\right)^{\frac{2}{p}} \\
& \quad \leq c .
\end{aligned}
$$

Similarly, if $\int_{\Omega} b(x)^{-\frac{2}{q-2}}(x) d x<\infty$, we have

$$
\begin{aligned}
& \iint_{Q_{T}}\left|\nabla u_{\varepsilon}\right|^{2} d x d t \\
& \quad=c \iint_{Q_{T}} b(x)^{-\frac{2}{q}} b(x)^{\frac{2}{q}}\left|\nabla u_{\varepsilon}\right|^{2} d x d t \\
& \quad \leq c\left(\iint_{Q_{T}} b(x)^{-\frac{2}{q-2}} d x d t\right)^{\frac{q-2}{q}}\left(\iint_{Q_{T}} b(x)\left|\nabla u_{\varepsilon}\right|^{q} d x d t\right)^{\frac{2}{q}} \\
& \quad \leq c .
\end{aligned}
$$

Thirdly, we have

$$
\begin{aligned}
\sum_{i=1}^{N} & \iint_{Q_{T}} u_{\varepsilon t} \frac{\partial f_{i}\left(u_{\varepsilon}, x, t\right)}{\partial x_{i}} d x d t \\
\leq & \sum_{i=1}^{N} \iint_{Q_{T}}\left|f_{i u_{\varepsilon}}\left(u_{\varepsilon}, x, t\right)\right|\left|u_{\varepsilon x_{i}}\right|\left|u_{\varepsilon t}\right| d x d t \\
& +\sum_{i=1}^{N}+\iint_{Q_{T}}\left|f_{i x_{i}}\left(u_{\varepsilon}, x, t\right)\right|\left|u_{\varepsilon t}\right| d x d t \\
\leq & \frac{1}{2} \iint_{Q_{T}}\left|u_{\varepsilon t}\right|^{2} d x d t+c \iint_{Q_{T}}\left|\nabla u_{\varepsilon}\right|^{2} d x d t+c .
\end{aligned}
$$

Combining inequalities (3.24)-(3.28) with (3.23), we can extrapolate that

$$
\begin{aligned}
& \iint_{Q_{T}}\left|u_{\varepsilon t}\right|^{2} d x d t+\iint_{Q_{T}}(a(x)+\varepsilon) \frac{d}{d t} \int_{0}^{\left|\nabla u_{\varepsilon}\right|^{2}+\varepsilon} s^{\frac{p-2}{2}} d s d x d t \\
& \quad+\iint_{Q_{T}} b(x) \frac{d}{d t} \int_{0}^{\left|\nabla u_{\varepsilon}\right|^{2}+\varepsilon} s^{\frac{q-2}{2}} d s d x d t \\
& \quad \leq c
\end{aligned}
$$


and so

$$
\begin{aligned}
& \iint_{Q_{T}}\left|u_{\varepsilon t}\right|^{2} d x d t \\
& \quad \leq c+c \int_{\Omega}(a(x)+\varepsilon) \int_{0}^{\left|\nabla u_{\varepsilon}\right|^{2}+\varepsilon} s^{\frac{p-2}{2}} d s d x \\
& \quad+c \int_{\Omega}(b(x)+\varepsilon) \int_{0}^{\left|\nabla u_{\varepsilon}\right|^{2}+\varepsilon} s^{\frac{q-2}{2}} d s d x \\
& \quad \leq c .
\end{aligned}
$$

According to the weak convergence theory, by (3.20), (3.21), (3.22), and (3.29), there exist a function $u$ and two $N$-dimensional vector functions $\vec{\zeta}=\left(\zeta_{1}, \ldots, \zeta_{N}\right)$ and $\vec{\xi}=\left(\xi_{1}, \ldots, \xi_{N}\right)$ such that

$$
\begin{aligned}
& u \in L^{\infty}\left(Q_{T}\right), \quad\left|\zeta_{i}\right| \in L^{\frac{p}{p-1}}\left(Q_{T}\right), \quad\left|\xi_{i}\right| \in L^{\frac{q}{q-1}}\left(Q_{T}\right), \\
& u_{\varepsilon} \rightarrow u, \quad \text { a.e. in } Q_{T}, \\
& u_{\varepsilon} \rightarrow * u, \quad \text { in } L^{\infty}\left(Q_{T}\right), \\
& f_{i}\left(u_{\varepsilon}, x, t\right) \rightarrow f_{i}(u, x, t), \quad \text { a.e. in } Q_{T}, \\
& a(x)\left|\nabla u_{\varepsilon}\right|^{p-2} u_{\varepsilon x_{i}} \rightarrow \zeta_{i}, \quad \text { in } L^{\frac{p}{p-1}}\left(Q_{T}\right), \\
& b(x)\left|\nabla u_{\varepsilon}\right|^{q-2} u_{\varepsilon x_{i}} \rightarrow \xi_{i}, \quad \text { in } L^{\frac{q}{q-1}}\left(Q_{T}\right) .
\end{aligned}
$$

At last, it is not difficult to show that

$$
\begin{aligned}
& \lim _{\varepsilon \rightarrow 0} \iint_{Q_{T}}(a(x)+\varepsilon)(a(x)+\varepsilon)\left(\left|\nabla u_{\varepsilon}\right|^{2}+\varepsilon\right)^{\frac{p-2}{2}}\left|\nabla u_{\varepsilon}\right|^{2} \nabla u_{\varepsilon} \nabla \varphi d x d t \\
& \quad+\lim _{\varepsilon \rightarrow 0} \iint_{Q_{T}}(b(x)+\varepsilon)(a(x)+\varepsilon)\left(\left|\nabla u_{\varepsilon}\right|^{2}+\varepsilon\right)^{\frac{q-2}{2}}\left|\nabla u_{\varepsilon}\right|^{2} \nabla u_{\varepsilon} \nabla \varphi d x d t \\
& \quad=\iint_{Q_{T}}(\vec{\zeta}+\bar{\xi}) \cdot \nabla \varphi d x d t \\
& \quad=\iint_{Q_{T}}\left[a(x)|\nabla u|^{p-2} \nabla u+b(x)|\nabla u|^{q-2} \nabla u\right] \nabla \varphi d x d t
\end{aligned}
$$

for any given $\varphi \in C_{0}^{1}\left(Q_{T}\right)$. So $u \in L^{p}\left(0, T ; W_{\text {loc }}^{1, p}(\Omega)\right) \cap L^{q}\left(0, T ; W_{\text {loc }}^{1, q}(\Omega)\right)$, and (2.6) is true.

In addition, we can choose the test function $\varphi(x, t)=\chi_{\left[t_{1}, t_{2}\right]} \phi(x)$ in which $\phi(x) \in C_{0}^{\infty}(\Omega)$ and $\chi_{\left[t_{1}, t_{2}\right]}$ is the characteristic function of $\left[t_{1}, t_{2}\right] \subset(0, T)$. Then

$$
\begin{aligned}
& \int_{t_{1}}^{t_{2}} \int_{\Omega}\left[\left(a(x)|\nabla u|^{p-2} \nabla u+b(x)|\nabla u|^{q-2} \nabla u\right) \nabla \phi+\sum_{i=1}^{N} f_{i}(x, t, u) \phi(x)\right] d x d t \\
& \quad=\int_{\Omega}\left(u\left(x, t_{2}\right)-u\left(x, t_{1}\right)\right) \phi(x) d x .
\end{aligned}
$$

Let $t=t_{2}$ and $t_{1} \rightarrow 0$. Then we have (2.7). Moreover, by the following proposition, $u$ can be defined as the trace on the boundary $\partial \Omega, u$ is a solution of equation (1.1) with the initial-boundary value conditions (1.7)-(1.8). Theorem 2.4 is proved. 
Proposition 3.3 If $u(x, t)$ is a weak solution of equation (1.1) with the initial value condition (1.4) and one of the following conditions is true:

(i)

$$
\int_{\Omega} a(x)^{-\frac{1}{p-1}} d x<\infty
$$

(ii)

$$
\int_{\Omega} b(x)^{-\frac{1}{q-1}} d x<\infty
$$

then

$$
\int_{\Omega}|\nabla u| d x \leq c(T)
$$

Proof If (i) is true, then

$$
\begin{aligned}
\int_{\Omega}|\nabla u| d x= & \int_{\left\{x \in \Omega: a(x)^{\frac{1}{p-1}}|\nabla u| \leq 1\right\}}|\nabla u| d x \\
& +\int_{\left\{x \in \Omega: a^{\frac{1}{p-1}}|\nabla u|>1\right\}}|\nabla u| d x \\
\leq & c \int_{\Omega} a(x)^{-\frac{1}{p-1}} d x+\int_{\Omega} a(x)|\nabla u|^{p} d x+c \\
\leq & c .
\end{aligned}
$$

Similarly, if (ii) is true, we also have (3.32).

\section{The stability of the initial-boundary value problem}

For small $\eta>0$, we introduce the following functions:

$$
S_{\eta}(s)=\int_{0}^{s} h_{\eta}(\tau) d \tau, \quad h_{\eta}(s)=\frac{2}{\eta}\left(1-\frac{|s|}{\eta}\right)_{+}, \quad H_{\eta}(s)=\int_{0}^{s} S_{\eta}(\tau) d \tau \text {. }
$$

Obviously, we have $\left|s h_{\eta}(s)\right| \leq 1$ and

$$
\lim _{\eta \rightarrow 0} S_{\eta}(s)=\operatorname{sgn} s, \quad \lim _{\eta \rightarrow 0} s h_{\eta}(s)=0, \quad \lim _{\eta \rightarrow 0} H_{\eta}(s)=|s|, \quad s \in(-\infty,+\infty) .
$$

Proposition 4.1 Let $u(x, t)$ and $v(x, t)$ be two solutions of equation (1.1) with the homogeneous value condition

$$
u(x, t)=v(x, t)=0, \quad(x, t) \in \partial \Omega \times(0, T)
$$

and with different initial values $u_{0}(x)$ and $v_{0}(x)$ respectively. If $p>1,(3.30)$ or (3.31) is true, and

$$
\left|f_{i}(u, x, t)-f_{i}(v, x, t)\right| \leq c\left[a(x)^{\frac{1}{p}}+b(x)^{\frac{1}{q}}\right]|u-v|, \quad i=1,2, \ldots, N
$$


then

$$
\int_{\Omega}|u(x, t)-v(x, t)| d x \leq \int_{\Omega}\left|u_{0}(x)-v_{0}(x)\right| d x, \quad t \in[0, T) .
$$

Proof Since (3.30) or (3.31) is true, Proposition 3.3 implies that the boundary value condition (4.2) is true in the sense of trace. Choose $\varphi=\chi_{[\tau, s]} S_{\eta}(u-v)$ as the test function, where $\chi_{[\tau, s]}$ is the characteristic function of $[\tau, s] \subset(0, T)$. Then

$$
\begin{aligned}
\int_{\tau}^{s} \int_{\Omega} & S_{\eta}(u-v) \frac{\partial(u-v)}{\partial t} d x d t \\
& +\int_{\tau}^{s} \int_{\Omega} a(x)\left(|\nabla u|^{p-2} \nabla u-|\nabla v|^{p-2} \nabla v\right) \nabla(u-v) h_{\eta}(u-v) d x d t \\
& +\int_{\tau}^{s} \int_{\Omega} b(x)\left(|\nabla u|^{q-2} \nabla u-|\nabla v|^{q-2} \nabla v\right) \nabla(u-v) h_{\eta}(u-v) d x d t \\
& \left.+\sum_{i=1}^{N} \int_{\tau}^{s} \int_{\Omega}\left[f_{i}(u, x, t)\right)-f_{i}(v, x, t)\right](u-v)_{x_{i}} h_{\eta}(u-v) d x d t \\
= & 0 .
\end{aligned}
$$

By that $\iint_{Q_{T}}\left|u_{t}\right| d x d t \leq c, \iint_{Q_{T}}\left|v_{t}\right| d x d t \leq c$, we can use the dominated convergence theorem to obtain

$$
\begin{aligned}
& \lim _{\eta \rightarrow 0} \int_{\tau}^{s} \int_{\Omega} S_{\eta}(u-v) \frac{\partial(u-v)}{\partial t} d x d t \\
& =\lim _{\eta \rightarrow 0} \int_{\Omega}\left[H_{\eta}(u-v)(x, s)-H_{\eta}(u-v)(x, \tau)\right] d x \\
& \quad=\int_{\Omega}|u-v|(x, s) d x-\int_{\Omega}|u-v|(x, \tau) d x
\end{aligned}
$$

Also, since a $p$-Laplacian operator is a monotone operator, then we have

$$
\int_{\tau}^{s} \int_{\Omega} a(x)\left(|\nabla u|^{p-2} \nabla u-|\nabla v|^{p-2} \nabla v\right) \nabla(u-v) h_{\eta}(u-v) d x d t \geq 0
$$

and

$$
\int_{\tau}^{s} \int_{\Omega} b(x)\left(|\nabla u|^{q-2} \nabla u-|\nabla v|^{q-2} \nabla v\right) \nabla(u-v) h_{\eta}(u-v) d x d t \geq 0 .
$$

Moreover, since $f_{i}(s, x, t)$ satisfies (4.3), we have

$$
\begin{aligned}
& \lim _{\eta \rightarrow 0} \sum_{i=1}^{N}\left|\int_{\tau}^{s} \int_{\Omega}\left[f_{i}(u, x, t)-f_{i}(v, x, t)\right](u-v)_{x_{i}} h_{\eta}(u-v) d x d t\right| \\
& \quad \leq c \lim _{\eta \rightarrow 0} \int_{\tau}^{s} \int_{\Omega}\left|h_{\eta}(u-v)(u-v)\right|\left[a(x)^{\frac{1}{p}}+b(x)^{\frac{1}{q}}\right]|\nabla(u-v)| d x d t \\
& \quad \leq c \lim _{\eta \rightarrow 0} \int_{\tau}^{s} \int_{\Omega}\left|h_{\eta}(u-v)(u-v)\right| a(x)^{\frac{1}{p}}|\nabla(u-v)| d x d t
\end{aligned}
$$




$$
\begin{aligned}
& +c \lim _{\eta \rightarrow 0} \int_{\tau}^{s} \int_{\Omega}\left|h_{\eta}(u-v)(u-v)\right| b(x)^{\frac{1}{q}}|\nabla(u-v)| d x d t \\
\leq & c \lim _{\eta \rightarrow 0}\left(\int_{\tau}^{s} \int_{\Omega} a(x)\left(|\nabla u|^{p}+|\nabla v|^{p}\right) d x d t\right)^{\frac{1}{p}} \\
& \cdot\left(\int_{\tau}^{s} \int_{\Omega}\left|(u-v) h_{\eta}(u-v)\right|^{\frac{p}{p-1}} d x d t\right)^{\frac{p-1}{p}} \\
& +c \lim _{\eta \rightarrow 0}\left(\int_{\tau}^{s} \int_{\Omega} b(x)\left(|\nabla u|^{q}+|\nabla v|^{q}\right) d x d t\right)^{\frac{1}{q}} \\
& \cdot\left(\int_{\tau}^{s} \int_{\Omega}\left|(u-v) h_{\eta}(u-v)\right|^{\frac{q}{q-1}} d x d t\right)^{\frac{q-1}{q}}
\end{aligned}
$$

$=0$.

Finally, let $\eta \rightarrow 0$ in (4.4). By (4.5)-(4.8), we have

$$
\int_{\Omega}|u(x, s)-v(x, s)| d x \leq \int_{\Omega}|u(x, \tau)-v(x, \tau)| d x .
$$

Let $\tau \rightarrow 0$. Then

$$
\int_{\Omega}|u(x, s)-v(x, s)| d x \leq \int_{\Omega}\left|u_{0}(x)-v_{0}(x)\right| d x .
$$

Proposition 4.1 is proved.

Proof of Theorem 2.5 Comparing with Proposition 4.1, Theorem 2.5 is not with condition (4.3). By checking the proof of Proposition 4.1, we only need to show

$$
\lim _{\eta \rightarrow 0}\left|\int_{\Omega}\left[f_{i}(u, x, t)-f_{i}(v, x, t)\right](u-v)_{x_{i}} h_{\eta}(u-v) d x\right|=0
$$

without condition (4.3). We give the details below

$$
\begin{aligned}
& \int_{\Omega}\left[f_{i}(u, x, t)-f_{i}(v, x, t)\right](u-v)_{x_{i}} h_{\eta}(u-v) d x \\
& \quad=\int_{\{\Omega:|u-v|<\eta\}}\left[f_{i}(u, x, t)-f_{i}(v, x, t)\right](u-v)_{x_{i}} h_{\eta}(u-v) d x .
\end{aligned}
$$

When the set $\{\Omega:|u-v|=0\}$ is with zero measure, since $\left|f_{i}(u, x, t)-f_{i}(v, x, t)\right| \leq c$, we have

$$
\begin{aligned}
& \lim _{\eta \rightarrow 0} \mid \int_{\{\Omega:|u-v|<\eta\}}\left[f_{i}(u, x, t)-f_{i}(v, x, t)\right](u-v)_{x_{i}} h_{\eta}(u-v) d x \mid \\
&= \frac{1}{2} \lim _{\eta \rightarrow 0} \mid \int_{\{\Omega:|u-v|<\eta\}}\left[f_{i}(u, x, t)-f_{i}(v, x, t)\right] \\
& \cdot\left[a(x)^{\frac{1}{p-1}} a(x)^{-\frac{1}{p-1}}+b(x)^{\frac{1}{q-1}} b(x)^{-\frac{1}{q-1}}\right](u-v)_{x_{i}} h_{\eta}(u-v) d x \mid \\
& \leq c\left(\int_{\{\Omega:|u-v|=0\}}\left(a(x)\left(|\nabla u|^{p}+\mid \nabla v^{p}\right) \mid\right)^{p} d x\right)^{\frac{1}{p}}\left(\int_{\Omega} a(x)^{-\frac{1}{p-1}} d x\right)^{\frac{p-1}{p}}
\end{aligned}
$$




$$
\begin{aligned}
& +c\left(\int_{\{\Omega:|u-v|=0\}}\left(b(x)\left(|\nabla u|^{q}+|\nabla v|^{q}\right)\right)^{q} d x\right)^{\frac{1}{q}}\left(\int_{\Omega} b(x)^{-\frac{1}{q-1}} d x\right)^{\frac{q-1}{q}} \\
= & 0 .
\end{aligned}
$$

When the set $\{\Omega:|u-v|=0\}$ is with a positive measure, by (2.13), $\left[a(x)^{-\frac{1}{p-1}}+b(x)^{-\frac{1}{p-1}}\right] \in$ $L^{1}(\Omega)$, we have

$$
\begin{aligned}
& \lim _{\eta \rightarrow 0}\left|\int_{\{\Omega:|u-v|<\eta\}}\left[f_{i}(u, x, t)-f_{i}(v, x, t)\right](u-v)_{x_{i}} h_{\eta}(u-v) d x\right| \\
& \leq c\left(\int_{\{\Omega:|u-v|=0\}}\left(a(x)^{\frac{1}{p}}|\nabla u-\nabla v|\right)^{p} d x\right)^{\frac{1}{p}}\left(\int_{\Omega} a(x)^{-\frac{1}{p-1}} d x\right)^{\frac{p-1}{p}} \\
& \quad+c\left(\int_{\{\Omega:|u-v|=0\}}\left(b(x)^{\frac{1}{q}}|\nabla u-\nabla v|\right)^{q} d x\right)^{\frac{1}{q}}\left(\int_{\Omega} b(x)^{-\frac{1}{q-1}} d x\right)^{\frac{q-1}{q}} \\
& =0 .
\end{aligned}
$$

Thus, we have the conclusion.

\section{Proof of Theorems 2.6}

Proof of Theorems 2.6 Since $a(x) b(x)=0, x \in \partial \Omega$, we can define $\Omega_{\eta}=\{x \in \Omega: a(x) b(x)>\eta\}$,

$$
\phi_{\eta}(x)= \begin{cases}1, & \text { if } x \in \Omega_{\eta}, \\ \frac{a(x) b(x)}{\eta}, & \text { if } x \in \Omega \backslash \Omega_{\eta},\end{cases}
$$

and choose $\chi_{[\tau, s]} \phi_{\eta}(x) S_{\eta}(u-v)$ as the test function. Thus

$$
\begin{aligned}
\int_{\tau}^{s} \int_{\Omega} & \phi_{\eta} S_{\eta}(u-v) \frac{\partial(u-v)}{\partial t} d x d t \\
& +\int_{\tau}^{s} \int_{\Omega} a(x)\left(|\nabla u|^{p-2} \nabla u-|\nabla v|^{p-2} \nabla v\right) \nabla(u-v) h_{\eta}(u-v) \phi_{\eta}(x) d x d t \\
& +\int_{\tau}^{s} \int_{\Omega} a(x)\left(|\nabla u|^{p-2} \nabla u-|\nabla v|^{p-2} \nabla v\right)(u-v) S_{\eta}(u-v) \nabla \phi_{\eta} d x d t \\
& +\int_{\tau}^{s} \int_{\Omega} b(x)\left(|\nabla u|^{q-2} \nabla u-|\nabla v|^{q-2} \nabla v\right) \nabla(u-v) h_{\eta}(u-v) \phi_{\eta}(x) d x d t \\
& +\int_{\tau}^{s} \int_{\Omega} b(x)\left(|\nabla u|^{q-2} \nabla u-|\nabla v|^{q-2} \nabla v\right)(u-v) S_{\eta}(u-v) \nabla \phi_{\eta} d x d t \\
& +\sum_{i=1}^{N} \int_{\tau}^{s} \int_{\Omega}\left[f_{i}(u, x, t)-f_{i}(v, x, t)\right] \phi_{\eta x_{i}} S_{\eta}(u-v) d x d t \\
& +\sum_{i=1}^{N} \int_{\tau}^{s} \int_{\Omega}\left[f_{i}(u, x, t)-f_{i}(v, x, t)\right](u-v)_{x_{i}} \phi_{\eta} h_{\eta}(u-v) d x d t \\
= & 0 .
\end{aligned}
$$


Chan Advances in Difference Equations

(2021) 2021:502

Page 21 of 32

At first, we have

$$
\begin{gathered}
\lim _{\eta \rightarrow 0} \int_{\tau}^{s} \int_{\Omega} \phi_{\eta}(x) S_{\eta}(u-v) \frac{\partial(u-v)}{\partial t} d x d t \\
=\lim _{\eta \rightarrow 0} \int_{\tau}^{s} \int_{\Omega} \frac{\partial\left[\phi_{\eta}(x) H_{\eta}(u-v)\right]}{\partial t} d x d t \\
\quad=\int_{\Omega}|u-v|(x, s) d x-\int_{\Omega}|u-v|(x, \tau) d x .
\end{gathered}
$$

Secondly, we have

$$
\int_{\Omega} a(x)\left(|\nabla u|^{p-2} \nabla u-|\nabla v|^{p-2} \nabla v\right) \nabla(u-v) h_{\eta}(u-v) \phi_{\eta}(x) d x \geq 0
$$

and

$$
\int_{\Omega} b(x)\left(|\nabla u|^{q-2} \nabla u-|\nabla v|^{q-2} \nabla v\right) \nabla(u-v) h_{\eta}(u-v) \phi_{\eta}(x) d x \geq 0 .
$$

Thirdly, we have

$$
\nabla \phi_{\eta}(x)= \begin{cases}0, & \text { if } x \in \Omega_{\eta}, \\ \frac{1}{\eta} \nabla(a(x) b(x)), & \text { if } x \in \Omega \backslash \Omega_{\eta},\end{cases}
$$

condition (2.17) yields

$$
\begin{aligned}
\left|\int_{\Omega} a(x)\left(|\nabla u|^{p-2} \nabla u-|\nabla v|^{p-2} \nabla v\right) \nabla \phi_{\eta} S_{\eta}(u-v) d x\right| \\
=\left|\int_{\Omega \backslash \Omega_{\eta}} a(x)\left(|\nabla u|^{p-2} \nabla u-|\nabla v|^{p-2} \nabla v\right) \nabla \phi_{\eta} S_{\eta}(u-v) d x\right| \\
\leq \frac{1}{\eta} \int_{\Omega \backslash \Omega_{\eta}} a(x)\left(|\nabla u|^{p-1}+|\nabla v|^{p-1}\right) \nabla(a(x) b(x)) S_{\eta}(u-v) \mid d x \\
\leq \frac{c}{\eta}\left(\int_{\Omega \backslash \Omega_{\eta}}\left[a(x)\left(|\nabla u|^{p}+|\nabla v|^{p}\right)\right] d x\right)^{\frac{p-1}{p}} \\
\quad \cdot\left(\int_{\Omega \backslash \Omega_{\eta}} a(x)|\nabla(a(x) b(x))|^{p} d x\right)^{\frac{1}{p}} \\
\leq c\left[\left(\int_{\Omega \backslash \Omega_{\eta}} a(x)|\nabla u|^{p} d x\right)^{\frac{p-1}{p}}+\left(\int_{\Omega \backslash \Omega_{\eta}} a(x)|\nabla v|^{p} d x\right)^{\frac{p-1}{p}}\right] \\
\quad \cdot\left[\left(\int_{\Omega \backslash \Omega_{\eta}} a(x)^{1-p}|\nabla a|^{p} d x\right)^{\frac{1}{p}}+\left(\int_{\Omega \backslash \Omega_{\eta}} a(x) b(x)^{-p}|\nabla b|^{p} d x\right)^{\frac{1}{p}}\right] \\
\leq c\left[\left(\int_{\Omega \backslash \Omega_{\eta}} a(x)|\nabla u|^{p} d x\right)^{\frac{p-1}{p}}+\left(\int_{\Omega \backslash \Omega_{\eta}} a(x)|\nabla v|^{p} d x\right)^{\frac{p-1}{p}}\right],
\end{aligned}
$$


which goes to zero as $\eta \rightarrow 0$. Similarly, by (2.18), we have

$$
\begin{aligned}
& \left|\int_{\Omega} b(x)\left(|\nabla u|^{q-2} \nabla u-|\nabla v|^{q-2} \nabla v\right) \nabla \phi_{\eta} S_{\eta}(u-v) d x\right| \\
& =\left|\int_{\Omega \backslash \Omega_{\eta}} b(x)\left(|\nabla u|^{q-2} \nabla u-|\nabla v|^{q-2} \nabla v\right) \nabla \phi_{\eta} S_{\eta}(u-v) d x\right| \\
& \leq \leq \frac{1}{\eta} \int_{\Omega \backslash \Omega_{\eta}} b(x)\left(|\nabla u|^{q-1}+|\nabla v|^{q-1}\right) \nabla(a(x) b(x)) S_{\eta}(u-v) \mid d x \\
& \leq \frac{c}{\eta}\left(\int_{\Omega \backslash \Omega_{\eta}}\left[b(x)\left(|\nabla u|^{q}+|\nabla v|^{q}\right)\right] d x\right)^{\frac{q-1}{q}}\left(\int_{\Omega \backslash \Omega_{\eta}} b(x)|\nabla(a(x) b(x))|^{q} d x\right)^{\frac{1}{q}} \\
& \leq c\left[\left(\int_{\Omega \backslash \Omega_{\eta}} b(x)|\nabla u|^{q} d x\right)^{\frac{q-1}{q}}+\left(\int_{\Omega \backslash \Omega_{\eta}} b(x)|\nabla v|^{q} d x\right)^{\frac{q-1}{q}}\right] \\
& \quad \cdot\left[\left(\int_{\Omega \backslash \Omega_{\eta}} b(x)^{1-q}|\nabla b|^{q} d x\right)^{\frac{1}{q}}+\left(\int_{\Omega \backslash \Omega_{\eta}} b(x) a(x)^{-q}|\nabla a|^{q} d x\right)^{\frac{1}{q}}\right] \\
& \leq c\left[\left(\int_{\Omega \backslash \Omega_{\eta}} b(x)|\nabla u|^{q} d x\right)^{\frac{q-1}{q}}+\left(\int_{\Omega \backslash \Omega_{\eta}} b(x)|\nabla v|^{q} d x\right)^{\frac{q-1}{q}}\right],
\end{aligned}
$$

which goes to zero as $\eta \rightarrow 0$.

Fourthly, since $u(x, t), v(x, t) \in L^{\infty}\left(Q_{T}\right)$, condition (2.17) yields

$$
\begin{aligned}
& \lim _{\eta \rightarrow 0}\left|\int_{\Omega}\left[f_{i}(u, x, t)-f_{i}(v, x, t)\right] \phi_{\eta x_{i}} S_{\eta}(u-v) d x\right| \\
& =\lim _{\eta \rightarrow 0}\left|\int_{\Omega \backslash \Omega_{\eta}}\left[f_{i}(u, x, t)-f_{i}(v, x, t)\right] \phi_{\eta x_{i}} S_{\eta}(u-v) d x\right| \\
& \leq \lim _{\eta \rightarrow 0} \frac{1}{\eta} \int_{\Omega \backslash \Omega_{\eta}}\left[a(x)^{\frac{1}{p}}+b(x)^{\frac{1}{q}}\right]\left|(a(x) b(x))_{x_{i}}\right|\left|S_{\eta}(u-v)(u-v)\right| d x \\
& \leq \lim _{\eta \rightarrow 0}\left(\int_{\Omega \backslash \Omega_{\eta}}\left(a(x)^{1-p}|\nabla a|^{p}+a(x) b(x)^{-p}|\nabla b|^{p}\right) d x\right)^{\frac{1}{p}} \\
& \quad \times\left(\int_{\Omega} \mid S_{\eta}(u-v)(u-v)^{\frac{p}{p-1}} d x\right)^{\frac{p-1}{p}} \\
& \quad+\lim _{\eta \rightarrow 0}\left(\int_{\Omega \backslash \Omega_{\eta}}\left(b(x)^{1-q}|\nabla b|^{q}+b(x) a(x)^{-q}|\nabla a|^{q}\right) d x\right)^{\frac{1}{q}} \\
& \quad \times\left(\int_{\Omega} \mid S_{\eta}(u-v)(u-v)^{\frac{q}{q-1}} d x\right)^{\frac{q-1}{q}} \\
& \leq c\left(\int_{\Omega}|u-v| d x\right)^{\frac{p-1}{p}}+c\left(\int_{\Omega}|u-v| d x\right)^{\frac{q-1}{q}} .
\end{aligned}
$$

Moreover, by condition (2.16), we have

$$
\lim _{\eta \rightarrow 0} \sum_{i=1}^{N} \int_{\tau}^{s} \int_{\Omega}\left[f_{i}(u, x, t)-f_{i}(v, x, t)\right](u-v)_{x_{i}} \phi_{\eta} h_{\eta}(u-v) d x d t=0 .
$$


Finally, let $\eta \rightarrow 0$ in (5.2). Then

$$
\int_{\Omega}|u(x, s)-v(x, s)| d x \leq \int_{\Omega}|u(x, \tau)-v(x, \tau)| d x+c\left(\int_{0}^{t} \int_{\Omega}|u-v| d x d t\right)^{k} .
$$

where $k<1$.

By the generalized Gronwall inequality [28], we can extrapolate

$$
\int_{\Omega}|u(x, s)-v(x, s)| d x \leq c \int_{\Omega}|u(x, \tau)-v(x, \tau)| d x .
$$

Letting $\tau \rightarrow 0$, we have the stability (2.15).

Corollary 5.1 Suppose that $a(x)$ and $b(x)$ satisfy condition (a) or condition (b), and satisfy (2.17)(2.18). Let $q \geq p>1, a(x), b(x) \in C^{1}(\bar{\Omega})$ satisfy (1.5), and when $\left|s_{1}\right|,\left|s_{2}\right| \leq c$, there is a nonnegative continuous function $g_{i}(x)$ such that

$$
\left|f_{i}(x)\left(s_{1}, x, t\right)-f_{i}\left(s_{2}, x, t\right)\right| \leq c g_{i}(x), \quad i=1,2, \ldots, N .
$$

If $u(x, t)$ and $v(x, t)$ are two solutions of $(1.1)$ with the initial values $u_{0}(x)$ and $v_{0}(x)$ respectively and with the homogeneous boundary value condition

$$
u(x, t)=v(x, t)=0, \quad(x, t)=\Sigma_{p q} \times(0, T),
$$

then stability (2.15) is true, where

$$
\Sigma_{p q}=\left\{x \in \partial \Omega: \sum_{i=1}^{N}(a(x) b(x))_{x_{i}} g_{i}(x)=0\right\} .
$$

Proof Similar to the proof of Theorem 2.6, we have (5.2)-(5.7). Since $a(x)$ and $b(x)$ satisfy condition (a) or condition (b), Proposition 3.3 means that the partial boundary value condition (5.11) is true in the classical sense of the trace. Then by condition (2.17) it yields

$$
\begin{aligned}
& \lim _{\eta \rightarrow 0}\left|\int_{\Omega} \sum_{i=1}^{N}\left[f_{i}(u, x, t)-f_{i}(v, x, t)\right] \phi_{\eta x_{i}} S_{\eta}(u-v) d x\right| \\
& \quad=\lim _{\eta \rightarrow 0}\left|\int_{\Omega \backslash \Omega_{\eta}} \sum_{i=1}^{N}\left[f_{i}(u, x, t)-f_{i}(v, x, t)\right] \phi_{\eta x_{i}} S_{\eta}(u-v) d x\right| \\
& \quad \leq c \lim _{\eta \rightarrow 0} \frac{1}{\eta} \int_{\Omega \backslash \Omega_{\eta}} \sum_{i=1}^{N}\left|(a(x) b(x))_{x_{i}} g_{i}(x)\right|\left|S_{\eta}(u-v)(u-v)\right| d x \\
& \quad=c \int_{\partial \Omega} \sum_{i=1}^{N}\left|(a(x) b(x))_{x_{i}} g_{i}(x)\right||u-v| d x \\
& \quad=0 .
\end{aligned}
$$

The remaining process of the proof can be completed as that of Theorem 2.6. 


\section{Proof of Theorems 2.7}

In this section, we use a similar method as that used in the proof of Theorem 2.6 to prove Theorem 2.7.

Proof of Theorem 2.7 Let $u(x, t)$ and $v(x, t)$ be two weak solutions of equation (1.1) with the initial values $u_{0}(x)$ and $v_{0}(x)$ respectively. Different from the proof of Theorem 2.6), $a(x)$ and $b(x)$ may satisfy $(2.22)$.

Since $a(x)=b(x)=0$ when $x \in \partial \Omega$, but $a(x)+b(x)>0$ when $x \in \Omega$, we define $D_{\eta}=\{x \in$ $\Omega: a(x)+b(x)>\eta\}$ and let

$$
\varphi_{\eta}(x)= \begin{cases}1, & \text { if } x \in D_{\eta}, \\ \frac{a(x)+b(x)}{\eta}, & \text { if } x \in \Omega \backslash D_{\eta} .\end{cases}
$$

By choosing $\chi_{[\tau, s]} \varphi_{\eta}(x) S_{\eta}(u-v)$ as the test function, we have

$$
\begin{aligned}
& \int_{\tau}^{s} \int_{\Omega} \varphi_{\eta} S_{\eta}(u-v) \frac{\partial(u-v)}{\partial t} d x d t \\
& \quad+\int_{\tau}^{s} \int_{\Omega} a(x)\left(|\nabla u|^{p-2} \nabla u-|\nabla v|^{p-2} \nabla v\right) \nabla(u-v) h_{\eta}(u-v) \varphi_{\eta}(x) d x d t \\
& \quad+\int_{\tau}^{s} \int_{\Omega} a(x)\left(|\nabla u|^{p-2} \nabla u-|\nabla v|^{p-2} \nabla v\right)(u-v) S_{\eta}(u-v) \nabla \varphi_{\eta} d x d t \\
& \quad+\int_{\tau}^{s} \int_{\Omega} b(x)\left(|\nabla u|^{q-2} \nabla u-|\nabla v|^{q-2} \nabla v\right) \nabla(u-v) h_{\eta}(u-v) \varphi_{\eta}(x) d x d t \\
& \quad+\int_{\tau}^{s} \int_{\Omega} b(x)\left(|\nabla u|^{q-2} \nabla u-|\nabla v|^{q-2} \nabla v\right)(u-v) S_{\eta}(u-v) \nabla \varphi_{\eta} d x d t \\
& \quad+\sum_{i=1}^{N} \int_{\tau}^{s} \int_{\Omega}\left[f_{i}(u, x, t)-f_{i}(v, x, t)\right] \varphi_{\eta x_{i}} S_{\eta}(u-v) d x d t \\
& \quad+\sum_{i=1}^{N} \int_{\tau}^{s} \int_{\Omega}\left[f_{i}(u, x, t)-f_{i}(v, x, t)\right](u-v)_{x_{i}} \varphi_{\eta} h_{\eta}(u-v) d x d t \\
& =0 .
\end{aligned}
$$

Directly, we have the following three formulas similar to (5.3)-(5.5):

$$
\begin{aligned}
& \lim _{\eta \rightarrow 0} \int_{\tau}^{s} \int_{\Omega} \varphi_{\eta}(x) S_{\eta}(u-v) \frac{\partial(u-v)}{\partial t} d x d t \\
& \quad=\lim _{\eta \rightarrow 0} \int_{\tau}^{s} \int_{\Omega} \frac{\partial\left[\varphi_{\eta}(x) H_{\eta}(u-v)\right]}{\partial t} d x d t \\
& \quad=\int_{\Omega}|u-v|(x, s) d x-\int_{\Omega}|u-v|(x, \tau) d x, \\
& \int_{\Omega} a(x)\left(|\nabla u|^{p-2} \nabla u-|\nabla v|^{p-2} \nabla v\right) \nabla(u-v) h_{\eta}(u-v) \varphi_{\eta}(x) d x \geq 0
\end{aligned}
$$

and

$$
\int_{\Omega} b(x)\left(|\nabla u|^{q-2} \nabla u-|\nabla v|^{q-2} \nabla v\right) \nabla(u-v) h_{\eta}(u-v) \varphi_{\eta}(x) d x \geq 0 .
$$


Moreover, condition (2.20) yields

$$
\begin{aligned}
& \left|\int_{\Omega} a(x)\left(|\nabla u|^{p-2} \nabla u-|\nabla v|^{p-2} \nabla v\right) \nabla \varphi_{\eta} S_{\eta}(u-v) d x\right| \\
& \quad=\left|\int_{\Omega \backslash D_{\eta}} a(x)\left(|\nabla u|^{p-2} \nabla u-|\nabla v|^{p-2} \nabla v\right) \nabla \varphi_{\eta} S_{\eta}(u-v) d x\right| \\
& \quad \leq \frac{1}{\eta} \int_{\Omega \backslash D_{\eta}} a(x)\left(|\nabla u|^{p-1}+|\nabla v|^{p-1}\right) \nabla(a(x)+b(x)) S_{\eta}(u-v) \mid d x \\
& \quad \leq \frac{c}{\eta}\left(\int_{\Omega \backslash D_{\eta}}\left[a(x)\left(|\nabla u|^{p}+|\nabla v|^{p}\right)\right] d x\right)^{\frac{p-1}{p}}\left(\int_{\Omega \backslash D_{\eta}} a(x)|\nabla(a(x)+b(x))|^{p} d x\right)^{\frac{1}{p}} \\
& \quad \leq c\left[\left(\int_{\Omega \backslash D_{\eta}} a(x)|\nabla u|^{p} d x\right)^{\frac{p-1}{p}}+\left(\int_{\Omega \backslash D_{\eta}} a(x)|\nabla v|^{p} d x\right)^{\frac{p-1}{p}}\right]
\end{aligned}
$$

which goes to zero as $\eta \rightarrow 0$. Similarly, by (2.14), we can show that

$$
\begin{aligned}
& \left|\int_{\Omega} b(x)\left(|\nabla u|^{q-2} \nabla u-|\nabla v|^{q-2} \nabla v\right) \nabla \varphi_{\eta} S_{\eta}(u-v) d x\right| \\
& \quad \leq c\left[\left(\int_{\Omega \backslash D_{\eta}} b(x)|\nabla u|^{q} d x\right)^{\frac{q-1}{q}}+\left(\int_{\Omega \backslash D_{\eta}} b(x)|\nabla v|^{q} d x\right)^{\frac{q-1}{q}}\right],
\end{aligned}
$$

which goes to zero as $\eta \rightarrow 0$.

At the same time, since $u(x, t), v(x, t) \in L^{\infty}\left(Q_{T}\right)$, condition (2.19) yields

$$
\begin{aligned}
\lim _{\eta \rightarrow 0}\left|\int_{\Omega}\left[f_{i}(u, x, t)-f_{i}(v, x, t)\right] \varphi_{\eta x_{i}} S_{\eta}(u-v) d x\right| \\
=\lim _{\eta \rightarrow 0}\left|\int_{\Omega \backslash D_{\eta}}\left[f_{i}(u, x, t)-f_{i}(v, x, t)\right] \varphi_{\eta x_{i}} S_{\eta}(u-v) d x\right| \\
\leq \lim _{\eta \rightarrow 0} \frac{1}{\eta} \int_{\Omega \backslash D_{\eta}}\left[(a(x)+b(x))^{\frac{1}{p}}+(a(x)+b(x))^{\frac{1}{q}}\right] \\
\quad \times\left|(a(x)+b(x))_{x_{i}}\right|\left|S_{\eta}(u-v)(u-v)\right| d x \\
\leq \lim _{\eta \rightarrow 0}\left(\int_{\Omega \backslash D_{\eta}}\left(|\nabla(a+b)|^{1+\frac{1}{p}}\right) d x\right)^{\frac{1}{p}}\left(\int_{\Omega}\left|S_{\eta}(u-v)(u-v)\right|^{\frac{p}{p-1}} d x\right)^{\frac{p-1}{p}} \\
\quad+\lim _{\eta \rightarrow 0}\left(\int_{\Omega \backslash D_{\eta}}\left(|\nabla(a+b)|^{1+\frac{1}{p}}\right) d x\right)^{\frac{1}{q}}\left(\int_{\Omega}\left|S_{\eta}(u-v)(u-v)\right|^{\frac{q}{q-1}} d x\right)^{\frac{q-1}{q}} \\
\leq c\left(\int_{\Omega}|u-v| d x\right)^{\frac{p-1}{p}}+c\left(\int_{\Omega}|u-v| d x\right)^{\frac{q-1}{q}} \cdot
\end{aligned}
$$

For another term on the left-hand side of (6.2), conditions (2.19)-(2.21) yield

$$
\lim _{\eta \rightarrow 0} \sum_{i=1}^{N} \int_{\tau}^{s} \int_{\Omega}\left[f_{i}(u, x, t)-f_{i}(v, x, t)\right](u-v)_{x_{i}} \varphi_{\eta} h_{\eta}(u-v) d x d t=0 .
$$


Finally, let $\eta \rightarrow 0$ in (6.2). Then

$$
\int_{\Omega}|u(x, s)-v(x, s)| d x \leq \int_{\Omega}|u(x, \tau)-v(x, \tau)| d x+c\left(\int_{0}^{t} \int_{\Omega}|u-v| d x d t\right)^{k} .
$$

where $k<1$.

Similar to the proof of Theorem 2.6, we can deduce conclusion (2.15).

Corollary 6.1 Suppose that $a(x)$ and $b(x)$ satisfy condition (a) or condition (b), and satisfy (2.20), (2.21). Let $q \geq p>1, a(x), b(x) \in C^{1}(\bar{\Omega})$ satisfy (1.4), and when $\left|s_{1}\right|,\left|s_{2}\right| \leq c, f_{i}(s, x, t)$ satisfies (5.10). If $u(x, t)$ and $v(x)$ are two solutions of (1.1) with the initial values $u_{0}(x)$ and $v_{0}(x)$ respectively and with the homogeneous boundary value condition

$$
u(x, t)=v(x, t)=0, \quad(x, t)=\Sigma_{p q} \times(0, T),
$$

then stability (2.15) is true, where

$$
\Sigma_{p q}=\left\{x \in \partial \Omega: \sum_{i=1}^{N}(a(x)+b(x))_{x_{i}} g_{i}(x)=0\right\} .
$$

The proof is similar to that of Corollary 5.1, we omit the details here.

\section{A generalization of trace}

Let $B V(\Omega)$ be the $B V$ function space, i.e., $\left|\frac{\partial f}{\partial x_{i}}\right|$ is a regular measure, and

$$
B V(\Omega)=\left\{f(x): \int_{\Omega}\left|\frac{\partial f}{\partial x_{i}}\right|<c, i=1,2, \ldots, N\right\} .
$$

Then $C_{0}^{\infty}(\Omega)$ is dense in $B V(\Omega)$, and so the trace of $f(x) \in B V(\Omega)$ on the boundary $\partial \Omega$ is defined as the limit of a sequence $f_{\varepsilon}(x)$ as

$$
\left.f(x)\right|_{x \in \partial \Omega}=\left.\lim _{\varepsilon \rightarrow 0} f_{\varepsilon}(x)\right|_{x \in \partial \Omega}
$$

It is well known that a BV function space is the weakest space such that integration by parts is still true.

For a degenerate parabolic equation, how to impose a suitable boundary condition has been an important and difficult problem for a long time. For example, if we consider the reaction-diffusion equation

$$
\frac{\partial u}{\partial t}=\operatorname{div}(a(u, x, t) \nabla u)+\operatorname{div}(b(u)), \quad(x, t) \in Q_{T},
$$

if $a(u, x, t)$ is smooth enough, then the weak solution $u(x, t) \in B V\left(Q_{T}\right)$ can be proved, and so one can impose the boundary value condition (1.8) in the sense of trace in the classical way [26, 30,32]. However, if $a(u, x, t)$ is just a continuous function or just a integral function, then one only can prove that there is a weak solution $u(x, t) \in L^{\infty}\left(Q_{T}\right)$, but $u(x, t)$ may not be a BV function. Equation (7.2) is of hyperbolic-parabolic mixed type. When $a \equiv 0$, equation (7.1) becomes a first-order hyperbolic equation, if the solution is merely 
in $L^{\infty}$, the author of [23] first extended the trace in a weaker sense by introducing an integral formulation of the boundary condition. [23]'s idea was generalized to deal with well-posedness of weak solutions to the strongly degenerate parabolic equations (7.2) in $[1,2,7,11,14,15,18,22]$.

In this paper, we first consider the evolutionary $p$-Laplacian equations of the form

$$
\frac{\partial u}{\partial t}-\operatorname{div}\left(\alpha(x)|\nabla u|^{p-2} \nabla u\right)-b_{i}(x) D_{i} u+c(x, t) u=f(x, t), \quad(x, t) \in Q_{T},
$$

where $D_{i}=\frac{\partial}{\partial x_{i}}, \alpha(x) \in C(\Omega), \alpha(x)>0$ in $\Omega$ but may be equal to 0 on the boundary $\partial \Omega$. The author of [25] classified the boundary $\partial \Omega$ into three parts: the nondegenerate boundary $\Sigma_{3}$, the weakly degenerate boundary $\Sigma_{4}$, and the strongly degenerate boundary $\Sigma^{0}$. In detail, the author of [25] denoted that

$$
\begin{aligned}
& \Sigma_{3}=\{x \in \partial \Omega: \alpha(x)>0\}, \\
& \Sigma_{4}=\left\{x \in \partial \Omega: \alpha(x)=0, \text { there exists } r>0, \text { such that } \int_{\Omega \cap B_{r}(x)} a(y)^{-\frac{1}{p-1}} d y<+\infty\right\}, \\
& \Sigma^{0}=\partial \Omega \backslash\left(\Sigma_{3} \cup \Sigma_{4}\right)=\left\{x \in \partial \Omega \text { : for any small } r>0, \int_{\Omega \cap B_{r}(x)} a(y)^{-\frac{1}{p-1}} d y=+\infty\right\},
\end{aligned}
$$

where $B_{r}(x)=\{y: d(x, y)<r\}$. Meanwhile, they defined

$$
\begin{aligned}
& \Sigma_{0}=\left\{x \in \Sigma^{0}: \sum_{i=1}^{N} b_{i}(x) n_{i}(x)=0\right\}, \\
& \Sigma_{1}=\left\{x \in \Sigma^{0}: \sum_{i=1}^{N} b_{i}(x) n_{i}(x)>0\right\}, \\
& \Sigma_{2}=\left\{x \in \Sigma^{0}: \sum_{i=1}^{N} b_{i}(x) n_{i}(x)<0\right\},
\end{aligned}
$$

where $\vec{n}=\left\{n_{i}(x)\right\}$ is the inner normal vector of $\partial \Omega$. In order to study the well-posedness of weak solutions to equation (7.3), they imposed a partial boundary value condition as

$$
u(x, t)=g(x, t),(x, t) \in\left(\Sigma_{2} \cup \Sigma_{3} \cup \Sigma_{4}\right) \times(0, T),
$$

where $g(x, t)$ is an appropriately smooth function.

According to Proposition 3.3, it is obvious that on $\left(\Sigma_{3} \cup \Sigma_{4}\right) \times(0, T)$ the boundary value condition is true in the classical trace sense. So, the trouble lies in that the classical trace of $u$ on the strongly degenerate boundary $\Sigma^{0}$ cannot be defined.

Denote that $\Omega_{\lambda}=\{x \in \Omega: d(x)>\lambda\}$ when $\lambda$ is a positive infinite variable, and denote by $\mathbf{B}$ the closure of the set $C_{0}^{\infty}\left(Q_{T}\right)$ with respect to the norm

$$
\|u\|_{\mathbf{B}}=\iint_{Q_{T}} a(x)\left(|u(x, t)|^{p}+|\nabla u(x, t)|^{p}\right) d x d t, \quad u \in \mathbf{B} .
$$


The author of [25] defined the trace of $u \in \mathbf{B}, u(x, t)=0$ on $\Sigma_{2}$ as

$$
\text { ess } \lim _{\lambda \rightarrow 0} \int_{\left\{x \in \partial \Omega_{\lambda}: \sum_{i=1}^{N} b_{i}(x) n_{i}(x)<0\right\}} u^{2} \sum_{i=1}^{N} b_{i}(x) n_{i}(x) d \sigma=0 .
$$

Remark 2.2 in [25] pointed out that the usual trace of $u \in \mathbf{B}, u(x, t)=0$ on $\Sigma_{3} \cup \Sigma_{4}$ also satisfies (7.5). So, (7.5) is a generalization of the usual trace of $u \in B V\left(Q_{T}\right)$ to that of $u \in \mathbf{B}$.

Moreover, we can generalize the trace of $u \in B V\left(Q_{T}\right)$ to that of $u \in \mathbf{B}$ by a more general way. Let $\phi(x)$ be a weak characteristic function of $\Omega$ [33], i.e., $\phi(x) \in C(\bar{\Omega}) \cap C^{1}\left(\Omega \backslash \Omega_{\mu}\right)$ and

$$
\phi(x)>0, \quad x \in \Omega
$$

where

$$
\Omega_{\mu}=\{x \in \Omega: \phi(x)>\mu\}
$$

In a very recent paper [34], using some idea of [25], we defined the trace of $u \in \mathbf{B}, u(x, t)=0$ on $\Sigma^{0}$ as

$$
\text { ess } \lim _{\mu \rightarrow 0^{+}} \int_{\left\{x \in \partial \Omega_{\mu}: \sum_{i=1}^{N} b_{i}(x) \phi_{i}(x)<0\right\}} u^{2} \sum_{i=1}^{N} b_{i}(x) \phi_{x_{i}}(x) d \sigma=0,
$$

and the partial boundary value condition matching up with equation (7.3) is

$$
u(x, t)=v(x, t)=0, \quad(x, t)=\Sigma_{p} \times(0, T)
$$

in the sense of (7.5), where

$$
\Sigma_{p}=\left\{x \in \partial \Omega: \sum_{i=1}^{N} b_{i}(x) \phi_{x_{i}}(x)<0\right\} .
$$

Secondly, let us come back to our main equation (1.1). Denote that

$$
\|u\|_{\mathbf{B}_{\mathbf{p}}}=\iint_{Q_{T}} a(x)\left(|u(x, t)|^{p}+|\nabla u(x, t)|^{p}\right) d x d t, \quad u \in \mathbf{B}_{\mathbf{p}},
$$

and

$$
\|u\|_{\mathbf{B}_{\mathbf{q}}}=\iint_{Q_{T}} b(x)\left(|u(x, t)|^{q}+|\nabla u(x, t)|^{q}\right) d x d t, \quad u \in \mathbf{B}_{\mathbf{q}} .
$$

If $a(x), b(x)$ satisfy (c), we cannot impose the boundary value condition (7.7) in the sense of the classical trace generally. However, inspired by [25,34], if $f_{i}$ satisfies (5.10), by checking the proof of Corollary 5.1, then we may generalize the trace of $u \in B V\left(Q_{T}\right)$ to that of $u \in \mathbf{B}_{\mathbf{p}} \cap \mathbf{B}_{\mathbf{q}}, u(x, t)=0$ as

$$
\operatorname{ess} \lim _{\lambda \rightarrow 0} \int_{\left\{x \in \partial \Omega_{\mu}: \sum_{i=1}^{N} g_{i}(x) \phi_{x_{i}} \neq 0\right\}}|u|\left|\sum_{i=1}^{N} g_{i}(x) \phi_{x_{i}}\right| d \sigma=0 .
$$


Accordingly, if $a(x), b(x)$ satisfy (c), in order to study the uniqueness of weak solution to equation (1.1), we can impose the partial boundary value condition

$$
u(x, t)=0, \quad(x, t) \in\left(\Sigma_{p} \cup \Sigma_{q}\right) \times(0, T),
$$

in the sense of (7.8), where

$$
\Sigma_{p}=\left\{x \in \partial \Omega: \text { for any small } r>0, \int_{\Omega \cap B_{r}(x)} a(y)^{-\frac{1}{p-1}} d y=+\infty\right\}
$$

and

$$
\Sigma_{q}=\left\{x \in \partial \Omega: \text { for any small } r>0, \int_{\Omega \cap B_{r}(x)} b(y)^{-\frac{1}{q-1}} d y=+\infty\right\} .
$$

Naturally, there are other ways to generalize the trace. For example, similar to [25, 34], one also can generalize the trace of $u \in B V\left(Q_{T}\right)$ to that of $u \in \mathbf{B}_{\mathbf{p}} \cap \mathbf{B}_{\mathbf{q}}, u(x, t)=0$ as

$$
\text { ess } \lim _{\lambda \rightarrow 0} \int_{\left\{x \in \partial \Omega_{\mu}: \sum_{i=1}^{N} g_{i}(x) \phi_{x_{i}} \neq 0\right\}}|u|^{2}\left|\sum_{i=1}^{N} g_{i}(x) \phi_{x_{i}}\right| d \sigma=0 \text {. }
$$

In this weak sense of trace, one also can study the stability of weak solution to equation (1.1) with the partial boundary value condition (7.9), provided $f_{i}$ satisfies

$$
\left|f_{i}(u, x, t)-f_{i}(v, x, t)\right| \leq g_{i}(x)|u-v|^{2} .
$$

The details are omitted here.

\section{About the regularity}

The following parabolic equation with $p, q$-growth

$$
u_{t}=\operatorname{div}\left(|\nabla u|^{p-2} \nabla u+|\nabla u|^{q-2} \nabla u\right), \quad(x, t) \in Q_{T},
$$

was studied in [8]. Actually, the main equation considered in [8] has a more general sense. The following definitions and theorems are deduced from [8] directly.

Definition 8.1 We identify a function $u \in L_{\text {loc }}^{q}\left(0, T ; W_{\mathrm{loc}}^{1, q}(\Omega)\right)$ as a weak solution of equation (8.1) if and only if

$$
\iint_{Q_{T}}\left[u \varphi_{t}-\left(|\nabla u|^{p-2} \nabla u+|\nabla u|^{q-2} \nabla u\right) \nabla \varphi\right] d x d t=0, \quad \forall \varphi \in C_{0}^{\infty}\left(Q_{T}\right)
$$

Theorem 8.2 (A priori estimate) Let $u \in L_{\mathrm{loc}}^{q}\left(0, T ; W_{\mathrm{loc}}^{1, q}(\Omega)\right)$ be a weaksolution of equation (8.1). Assume that

$$
2 \leq p \leq q<p+\frac{4}{N}
$$


Then we have $\nabla u \in L^{\infty}\left(Q_{T}, \mathbb{R}^{N}\right)$, and for any parabolic cylinder $Q_{\rho}\left(z_{0}\right) \subset \subset Q_{T}$ and $s \in$ $(0,1)$, there holds

$$
\sup _{Q_{s \rho}\left(z_{0}\right)}|\nabla u| \leq c\left[\frac{1}{[(1-s) \rho]^{\hat{n}+2}} \iint_{Q_{\rho}\left(z_{0}\right)}\left(1+|\nabla u|^{2}\right)^{\frac{q}{2}} d z\right]^{\frac{1}{q} \frac{2 q}{4-\hat{n} q-p}}
$$

for a constant $c$ which goes to $\infty$ as $q \rightarrow p+\frac{4}{N}$, where $\hat{n}=n$ if $n \geq 3, \hat{n}=$ any number $\epsilon$ $\left(2, \frac{4}{q-p}\right)$ if $n=2$.

Definition 8.3 We identify a function

$$
u \in L^{p}\left(0, T ; W_{0}^{1, p}(\Omega)\right) \cap L_{\mathrm{loc}}^{q}\left(0, T ; W_{\mathrm{loc}}^{1, q}(\Omega)\right)
$$

as a weak solution of the Cauchy-Dirichlet problem to equation (8.1) if and only if (8.2) holds and, moreover, the homogeneous Dirichlet boundary value condition is true in the sense of trace, the initial value condition is true in the sense

$$
\lim _{h \rightarrow 0} \frac{1}{h} \int_{0}^{h} \int_{\Omega}\left|u(x, t)-u_{0}(x)\right|^{2} d x d t=0 .
$$

By considering the Cauchy-Dirichlet problem to the following equation related to the $q$-Laplacian

$$
u_{t}=\operatorname{div}\left(|\nabla u|^{p-2} \nabla u+\left(|\nabla u|^{2}+\varepsilon\right)^{\frac{q-2}{2}} \nabla u\right), \quad(x, t) \in Q_{T},
$$

according to [17], there is a unique weak solution $u_{\varepsilon} \in C^{0}\left([0, T] ; L^{2}(\Omega)\right) \cap L^{q}\left(0, T ; W_{0}^{1, q}(\Omega)\right)$ with $\partial_{t} u_{\varepsilon} \in L^{q^{\prime}}\left(0, T ; W^{-1, q^{\prime}}(\Omega)\right)$. Based on this fact, using the Morse iterative technique and by the Stekov mean value method, the author of [8] proved the following theorem.

Theorem 8.4 (Existence of weak solutions) Suppose that

$$
2 \leq p \leq q<p+\frac{4}{N+2}
$$

holds. Then there exists a weak solution $u$ of the Cauchy-Dirichlet problem to equation (8.1). Moreover, its $L^{p}\left(0, T ; W_{0}^{1, p}(\Omega)\right)$ norm bounded by a constant depends only on $p, q$, $N,\left|u_{0}\right|_{L^{\infty}(\Omega)}$, and $\left|\nabla u_{0}\right|_{L^{r}(\Omega)}$. Further, the solution $u$ satisfies $\nabla u \in L_{\mathrm{loc}}^{\infty}\left(Q_{T}, \mathbb{R}^{N}\right)$ and $u_{t} \in$ $L^{\frac{q}{q-1}}\left(0, T ; W^{-1, \frac{p}{q-1}}(\Omega)\right)$. Once more, for any parabolic cylinder $Q_{\rho}\left(z_{0}\right) \subset \subset Q_{T}$ and $s \in(0,1)$, there holds

$$
\sup _{Q_{s \rho}\left(z_{0}\right)}|\nabla u| \leq c\left[\frac{1}{[(1-s) \rho]^{\hat{n}+2}} \iint_{Q_{\rho}\left(z_{0}\right)}\left(1+|\nabla u|^{2}\right)^{\frac{q}{2}} d z\right]^{\frac{1}{p} \frac{2 q}{4-(\hat{n}+2) q-p}},
$$

where $\hat{n}=n$ if $n \geq 3, \hat{n}=$ any number $\in\left(2, \frac{4}{q-p}-2\right)$ if $n=2$.

Recalling the main equation considered in this paper

$$
u_{t}=\operatorname{div}\left(a(x)|\nabla u|^{p-2} \nabla u+b(x)|\nabla u|^{q-2} \nabla u\right)+\sum_{i=1}^{N} \frac{\partial f_{i}(u, x, t)}{\partial x_{i}}, \quad(x, t) \in Q_{T},
$$


with that $a(x)>0$ and $b(x)>0$ when $x \in \Omega$. Since $f_{i}(s, x, t)$ is a $C^{1}$ function on $\mathbb{R} \times \bar{Q}_{T}$, $i=1,2, \ldots, N$, if we notice that the estimates about $|\nabla u|$ are local in Theorems 8.2 and 8.4, besides the existence theorem (Theorem 2.4 in Sect. 2), under conditions (8.3) and (8.6), then we conjecture that estimates (8.4) and (8.7) are true correspondingly. We are ready to discuss this problem thoroughly in the future; in particular, we are concerned with the boundary estimates about the weak solution $u(x, t)$ and the estimate of its gradient $|\nabla u|$ near the boundary.

\section{Acknowledgements}

The author would like to express his sincere thanks to the reviewers and the editors.

\section{Funding}

The paper is supported by NSF Fujian province, China (no: 2019J01858).

Availability of data and materials

No applicable.

\section{Declarations}

\section{Competing interests}

The author declares that he has no competing interests.

\section{Authors' contributions}

The author read and approved the final manuscript.

\section{Publisher's Note}

Springer Nature remains neutral with regard to jurisdictional claims in published maps and institutional affiliations.

Received: 2 February 2021 Accepted: 4 November 2021 Published online: 21 November 2021

\section{References}

1. Andreianov, B.P., Bendahmane, M., Karlsen, K.H.: Discrete duality finite volume schemes for doubly nonlinear degenerate hyperbolic-parabolic equations. http://www.math.ntnu.no/conservation/2009/001.html. Preprint

2. Andreianov, B.P., Bendahmane, M., Karlsen, K.H., Ouaro, S.: Well-posedness results for triply nonlinear degenerate parabolic equations. J. Differ. Equ. 247, 277-302 (2009)

3. Antontsev, S., Diaz, J., Shmarev, S.: Energy Methods for Free Boundary Problems, Applications to Nonlinear PDES and Fluid Mechanics, Progress in Nonlinear Differential Equations and Their Applications, vol. 48. Birkhäuser, Boston (2002)

4. Baroni, P., Colombo, M., Mingione, G.: Harnack inequalities for double phase functionals. Nonlinear Anal. 121, 206-222 (2015)

5. Baroni, P., Colombo, M., Mingione, G.: Nonautonomous functionals, borderline cases and related function classes. Algebra Anal. 27, 6-50 (2015) translation in St. Petersburg Math. J. 27, 347-379 (2016)

6. Baroni, P., Colombo, M., Mingione, G.: Regularity for general functionals with double phase. Calc. Var. Partial Differ. Equ. 57, 57-62 (2018)

7. Bendahmane, B., Karlsen, K.H.: Uniqueness of entropy solutions for quasilinear anisotropic degenerate parabolic equation. Contemp. Math. 371, 1-27 (2005)

8. Bögelein, V., Duzaar, F., Marcellini, P.: Parabolic equations with p, q-growth. J. Math. Pures Appl. 100, 535-563 (2013)

9. Bögelein, V., Duzaar, F., Marcellini, P.: Parabolic systems with p, q-growth: a variational approach. Arch. Ration. Mech. Anal. 210, 219-267 (2013)

10. Bögelein, V., Duzaar, F., Marcellini, P.: Existence of evolutionary variational solutions via the calculus of variations. J. Differ. Equ. 256, 3912-3942 (2014)

11. Carrillo, J.: Entropy solutions for nonlinear degenerate problems. Arch. Ration. Mech. Anal. 147, 269-361 (1999)

12. Diaz, J., Padial, J.: Uniqueness and existence of a solution in $B V_{t}(q)$ space to a doubly nonlinear parabolic problem. Publ. Mat. 40, 527-560 (1996)

13. Diaz, J., Thelin, F.: On a nonlinear parabolic problem arising in some models related to turbulent flows. SIAM J. Math Anal. 25, 1085-1111 (1994)

14. Kobayasi, K., Ohwa, H.: Uniqueness and existence for anisotropic degenerate parabolic equations with boundary conditions on a bounded rectangle. J. Differ. Equ. 252, 137-167 (2012)

15. Li, Y., Wang, Q.: Homogeneous Dirichlet problems for quasilinear anisotropic degenerate parabolic-hyperbolic equations. J. Differ. Equ. 252, 4719-4741 (2012)

16. Li, Z., Yan, B., Gao, W.: Existence of solutions to a parabolic $p(x)$-Laplace equation with convection term via $L^{\infty}$-esitmates. Electron. J. Differ. Equ. 2015, 46 (2015)

17. Lions, J.L.: Quelques méthodes de résolution des problémes aux limites non linéaries. Dunod, Paris (1969)

18. Lions, P.L., Perthame, B., Tadmor, E.: A kinetic formation of multidimensional conservation laws and related equations. J. Am. Math. Soc. 7, 169-191 (1994) 
19. Liu, Z: On the solvability of degenerate quasilinear parabolic equations of second order. Acta Math. Sin. 44, 77-84 (2001) (in Chinese)

20. Marcellini, P.: A variational approach to parabolic equations under general and p, q-growth conditions. Nonlinear Anal. 194, 111456 (2020)

21. Mascia, C., Porretta, A., Terracina, A.: Nonhomogeneous Dirichlet problems for degenerate parabolic-hyperbolic equations. Arch. Ration. Mech. Anal. 163, 87-124 (2002)

22. Michel, A., Vovelle, J.: Entropy formulation for parabolic degenerate equations with general Dirichlet boundary conditions and application to the convergence of FV methods. SIAM J. Numer. Anal. 41, 2262-2293 (2003)

23. Otto, A.: Initial-boundary value problem for a scalar conservation law. C. R. Acad. Sci., Sér. 1 Math. 322, 729-734 (1996)

24. Wu, Z., Zhao, J., Yin, J., Li, H.: Nonlinear Diffusion Equations. World Scientific, Singapore (2001)

25. Yin, J., Wang, C.: Evolutionary weighted $p$-Laplacian with boundary degeneracy. J. Differ. Equ. 237, 421-445 (2007)

26. Zhan, H.: The solution of a hyperbolic-parabolic mixed type equation on half space domain. J. Differ. Equ. 259, 1449-1481 (2015)

27. Zhan, H.: Existence of solutions to an evolution $p$-Laplacian equation with a nonlinear gradient term. Electron. J. Differ. Equ. 2017, 311 (2017)

28. Zhan, H.: On anisotropic parabolic equations with a nonlinear convection term depending on the spatial variable. Adv. Differ. Equ. 2019, 27 (2019)

29. Zhan, H.: The uniqueness of the solution to the diffusion equation with a damping term. Appl. Anal. 98, 1333-1346 (2019)

30. Zhan, H., Feng, Z:: A hyperbolic-parabolic mixed type equation with non-homogeneous boundary condition. J. Differ. Equ. 264, 7384-7411 (2018)

31. Zhan, H., Feng, Z: The stability of the solutions of convection diffusion equation. Nonlinear Anal. 182, 193-208 (2019)

32. Zhan, H., Feng, Z.: Partial boundary value condition for a degenerate parabolic equation. J. Differ. Equ. 267, 2874-2890 (2019)

33. Zhan, H., Feng, Z.: The well-posedness problem of an anisotropic parabolic equation. J. Differ. Equ. 268, 389-413 (2020)

34. Zhan, H., Feng, Z.: Optimal partial boundary condition for degenerate parabolic equations. J. Differ. Equ. 184, 156-182 (2021)

35. Zhang, Q., Radulescu, V.D.: Double phase anisotropic variational problems and combined effects of reaction and absorption terms. J. Math. Pures Appl. 118, 159-203 (2018)

\section{Submit your manuscript to a SpringerOpen ${ }^{\circ}$ journal and benefit from:}

- Convenient online submission

- Rigorous peer review

- Open access: articles freely available online

- High visibility within the field

- Retaining the copyright to your article

Submit your next manuscript at $\boldsymbol{\nabla}$ springeropen.com 